\title{
Effects of normal stress variation on the strength and stability of creeping faults
}

\author{
M. S. Boettcher \\ Marine Geology and Geophysics, Massachusetts Institute of Technology/Woods Hole Oceanographic Institution Joint \\ Program, Woods Hole Oceanographic Institution, Woods Hole, Massachusetts, USA \\ C. Marone \\ Department of Geosciences, Pennsylvania State University, University Park, Pennsylvania, USA \\ Received 2 October 2003; revised 30 December 2003; accepted 6 January 2004; published 11 March 2004.
}

[1] A central problem in studies of fault interaction and earthquake triggering is that of quantifying changes in frictional strength and the constitutive response caused by dynamic stressing. We imposed normal stress vibrations on creeping laboratory shear zones to investigate the process of dynamic weakening and the conditions under which resonant frictional behavior occurs. Layers of quartz powder were sheared at room temperature in a double-direct shear geometry at normal stress $\bar{\sigma}_{n}=25-200 \mathrm{MPa}$, vibration amplitude $A=$ $0.1-10 \mathrm{MPa}$, period $T=0.1-200 \mathrm{~s}$, and loading rate $V=1-1000 \mu \mathrm{m} / \mathrm{s}$. Frictional response varied systematically with $A, T$, and $V$. Small-amplitude, short-period vibrations had no effect on frictional strength, but large-amplitude, short-period vibrations reduced shear zone strength by about $1 \%$. Intermediate periods caused phase lags between shear strength and imposed vibrations. During long-period vibrations, frictional strength varied sinusoidally, in phase with vibrations and with an amplitude consistent with a constant coefficient of friction. Our data show that friction exhibits a critical vibration period, as predicted by theory. At long periods, the Dieterich (aging) friction law, with the Linker and Dieterich modification to describe step changes in normal stress, provides a good fit to our experimental results for all $A$ and $V$. At short periods, however, theory predicts more dynamic weakening than we observed experimentally, suggesting that existing rate and state friction laws do not account for the full physics of our laboratory experiments. Our data show that normal-force vibrations can weaken and potentially destabilize steadily creeping fault zones. INDEX TERMS: 7209 Seismology: Earthquake dynamics and mechanics; 8123 Tectonophysics: Dynamics, seismotectonics; 8168 Tectonophysics: Stresses—general; KEYWORDS: friction, earthquakes, vibrations

Citation: Boettcher, M. S., and C. Marone (2004), Effects of normal stress variation on the strength and stability of creeping faults, J. Geophys. Res., 109, B03406, doi:10.1029/2003JB002824.

\section{Introduction}

[2] Natural fault zones are continuously subject to variations in normal stress, affecting both their strength and stability. Earthquakes alter the static stress field surrounding their rupture and send transient and potentially destabilizing seismic waves thousands of kilometers into the distance. For example, dynamic stress changes from the $1992 M_{\mathrm{W}} 7.3$ Landers, California earthquake are known to have increased the seismicity rates both near [Gomberg et al., 1997; Wyss and Wiemer, 2000; Kilb et al., 2000, 2002] and far from the fault rupture [Hill et al., 1993; Gomberg and Bodin, 1994; Spudich et al., 1995; Gomberg, 1996; Gomberg and Davis, 1996; Gomberg et al., 1997; Harris, 1998], with the most aftershocks in the direction of rupture propagation where the dynamic stresses were largest [Hill et al., 1993; Kilb et

Copyright 2004 by the American Geophysical Union. 0148-0227/04/2003JB002824\$09.00 al., 2000, 2002]. The strength of the Landers fault zone itself increased continuously from 1992 to 1999 until shaking from the nearby $M_{W} 7.1$ Hector Mine earthquake disrupted the healing process [Vidale and $L i, 2003$ ]. Periodic stressing from ocean and Earth tides also alter stress on faults [Brodsky et al., 2003; Scholz, 2003]. Small-amplitude tidal stressing of the solid Earth did not effect seismicity rates in Southern California [Vidale et al., 1998], but both Wilcock [2001] and Tolstoy et al. [2002] observed correlations between the larger-amplitude ocean tidal cycles and microseismicity rates on the Juan de Fuca Ridge.

[3] Much insight into the process of dynamic earthquake triggering has come from previous laboratory experiments and numerical simulations. Laboratory work of Lockner and Beeler [1999] and Beeler and Lockner [2003] showed that triggering of stick-slip events is both amplitude- and frequency-dependent. A threshold nucleation time was observed, and it was found that only large-amplitude stress perturbations triggered seismicity when the period of the 


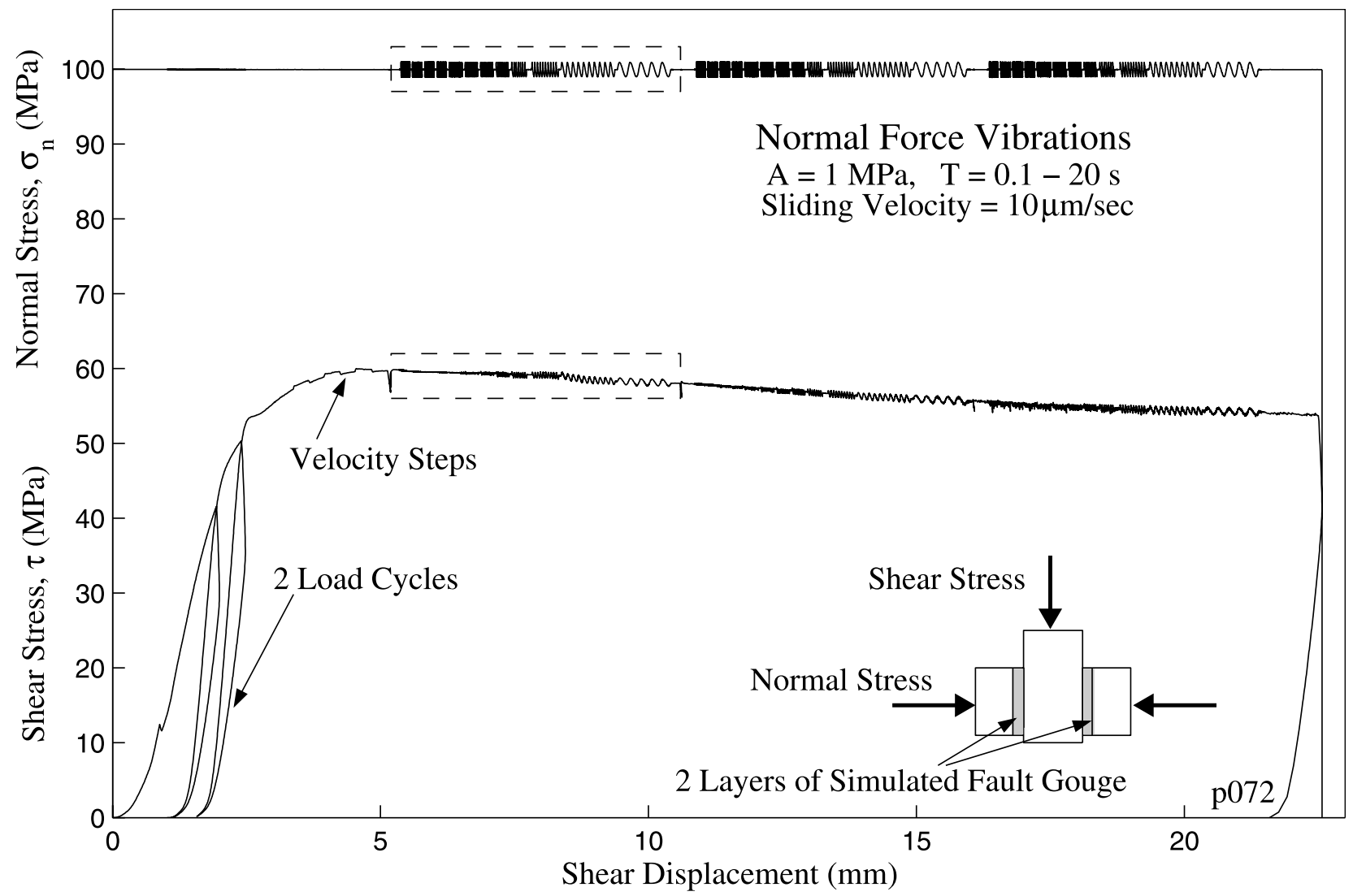

Figure 1. Imposed normal stress $\left(\sigma_{n}\right)$ and resultant frictional strength $(\tau)$ of quartz powder during a full experimental run. The inset shows double-direct shear geometry. The central block was driven at a rate $(V)$ of $10 \mu \mathrm{m} / \mathrm{s}$, under a mean normal load $\left(\bar{\sigma}_{n}\right)$ of $100 \mathrm{MPa}$, to a shear strain of 15 . Sinusoidal $\sigma_{n}$ vibrations, with an amplitude $(A)$ of $1 \mathrm{MPa}$ and a range of periods $(T)$, were preceded by two load cycles and six velocity steps $(10-20 \mu \mathrm{m} / \mathrm{s})$. The number in the bottom right (shown here and in the figures to follow) refers to the experiment number (Table 1). Dashed boxes indicate data shown in Figure 2.

perturbations was shorter than the nucleation time [Beeler and Lockner, 2003]. Furthermore, the results of Lockner and Beeler [1999] and Beeler and Lockner [2003] are consistent with observed earthquake rates in Southern California: higher-frequency, moderate- to large-amplitude shaking from the Landers earthquake triggered seismicity, and long-period, low-amplitude, tidal stressing did not.

[4] Tworzydlo and Hamzeh [1997] note that the inclusion of normal-force vibrations in models of rock friction can cause stably sliding simulations to go unstable. The work of Voisin [2001, 2002] shows that dynamic stressing of finite fault models, which obey either linear or nonlinear slipdependent friction laws, can result in a stability/instability transition and may trigger earthquakes. Normal-force vibrations increased the healing rate of Richardson and Marone's [1999] laboratory shear zones, which when combined with the observations of Vidale and $\mathrm{Li}$ [2003] indicate that stress changes can both increase and decrease the rate of fault zone strengthening. Theoretical work of Perfettini et al. [2001] suggest a resonant response of shear zone strength, involving strong amplification of the shear stress and velocity response for a small range of friction parameters and a critical loading stiffness.

[5] Normal stress variation may also play an important role in rupture propagation. On the basis of early thoughts on acoustic fluidization by Melosh [1979], Heaton [1990] suggested intense compressional waves could locally decrease the confining pressure ahead of a slip pulse. In the laboratory experiments of Bodin et al. [1998] a dynamic reduction in normal stress was observed during stick-slip events. Because normal stress should couple to slip anytime a fault has nonplanar geometry or nonhomogeneous materials, changes in normal stress are likely to be integral to both earthquake nucleation and rupture propagation.

[6] Rate and state friction laws, which have successfully modeled frictional sliding and earthquake phenomena for over two decades, were originally developed for constant normal stress conditions. In one formulation, termed the Dieterich or aging law, friction evolves during stationary contact [Dieterich, 1978, 1979], while in another, the Ruina or slip law, friction will only change with slip [Ruina, 1983]. These micromechanically distinct constitutive laws show macroscopically similar behavior under constant normal stress, but predict significantly different shear strength behavior when the steady state conditions are perturbed. Thus Perfettini et al. [2001] suggested laboratory experiments subject to normal stress vibrations to probe various formulations of the friction law.

[7] In this paper we report on laboratory experiments designed to investigate the effect of normal stress vibrations 

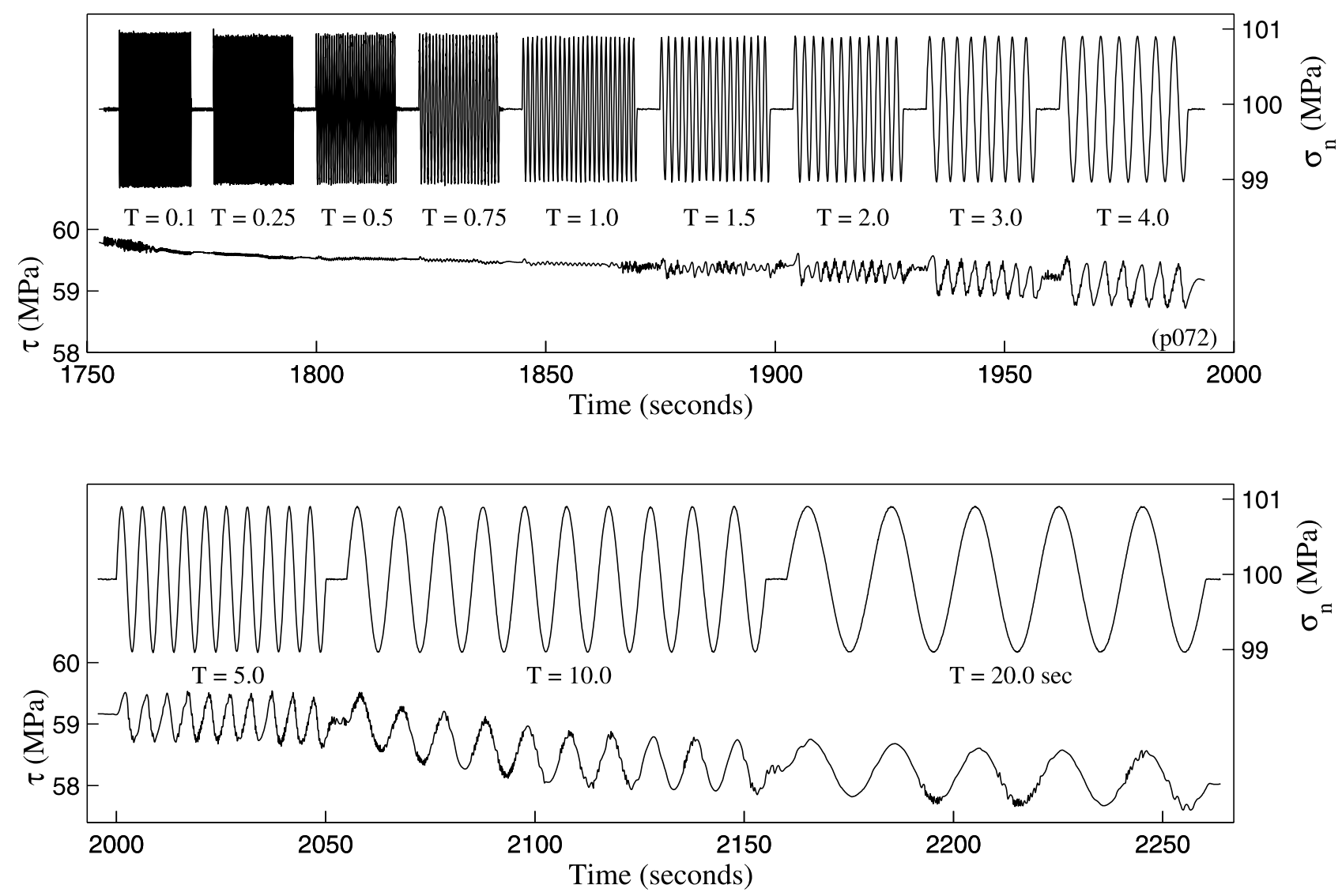

Figure 2. Frictional response $(\tau)$ of quartz powder to imposed normal stress $\left(\sigma_{n}\right)$ vibrations during steady creep. $\tau$ oscillations increased in amplitude with vibration period $(T)$. The character of $\tau$ oscillations evolved from no response at the shortest periods tested, to small-amplitude, sawtooth-shaped oscillations, to large-amplitude, symmetric sinusoids at the longest periods. The data are from the first vibration set of Figure 1 (dashed boxes).

on the strength and stability of steadily creeping shear zones. We show that shear zone strength varies systematically with vibration period, amplitude, and loading rate, in some cases resulting in dynamic fault weakening. We investigate the ability of the Dieterich and Ruina rate/state friction laws to model our laboratory observations and find that the Dieterich law provides the best fit.

\section{Experimental Procedure}

[8] Experiments were conducted in a biaxial loading apparatus at room temperature using double-direct shear geometry (inset to Figure 1) [see Mair and Marone, 1999; Karner and Marone, 2001; Frye and Marone, 2002]. In each experiment two, 3-mm-thick layers of quartz powder (U.S. Silica Co. F-110) were sandwiched between three steel forcing blocks. The two outer blocks have dimensions of $5.25 \times 5.0 \times 2.5 \mathrm{~cm}^{3}$ and the central block measures $7.5 \times 5.25 \times 3.75 \mathrm{~cm}^{3}$. The central block is longer than the side blocks and thus the nominal area of contact is constant during sliding. All blocks have grooves perpendicular to shear that constrain sliding to within the gouge layer, rather than along the gouge/steel interface.

[9] Vertical motion of the central forcing block was servo-controlled by displacement feedback and horizontal stress was maintained with constant load feedback. The force applied to each block as well as its position was measured and recorded by displacement transducers and load cells mounted on the driving rams just above the forcing blocks. Gouge layer thickness was calculated from displacement of the horizontal ram using the calibrated apparatus stiffness and elastic corrections. In some experiments, three Belleville disc springs were inserted into the standard load column to reduce the system stiffness from its intrinsic value of $k=0.5 \mathrm{KN} / \mu \mathrm{m}$. Together the springs are rated to support $300 \mathrm{KN}$, which is the shear force necessary for sliding at $57 \mathrm{MPa}$ normal stress. The springs were therefore most effective at low loads, reducing $k$ to 0.041 , 0.047 , and $0.052 \mathrm{KN} / \mu \mathrm{m}$ at normal stresses of 25,50 , and $100 \mathrm{MPa}$, respectively.

[10] Significant shear strain is required to obtain steady state frictional behavior in laboratory experiments [e.g., Beeler et al., 1996; Mair and Marone, 1999]. To reduce the net displacement needed to establish steady state shear zone fabric, and thus steady state frictional behavior [Marone, 1998], we began each experiment with load cycles and velocity steps following the procedure of Frye and Marone [2002] (Figure 1).

[11] We ran experiments at a constant load point velocity $V$ in the range $1-1000 \mu \mathrm{m} / \mathrm{s}$ and a $\bar{\sigma}_{n}$ between 25 and $200 \mathrm{MPa}$ (Table 1). Sinusoidal oscillations of the normal load were imposed on the sample by summing on 
Table 1. Experimental Parameters

\begin{tabular}{|c|c|c|c|c|}
\hline Experiment $^{\mathrm{a}}$ & $\sigma_{n}, \mathrm{MPa}$ & $T, \mathrm{~s}$ & $A, \mathrm{MPa}$ & $V, \mu \mathrm{m} / \mathrm{s}$ \\
\hline $\mathrm{m} 479$ & 25 & $0.1-10$ & $1,2,3$ & 10 \\
\hline $\mathrm{m} 480$ & 25,35 & $0.1-10$ & 2,3 & 10 \\
\hline $\mathrm{m} 481$ & 25,35 & $0.1-10$ & 2,3 & 10 \\
\hline $\mathrm{m} 484$ & 25,100 & $0.1-10$ & 4,10 & 10 \\
\hline m485 & 25,200 & $0.1-10$ & 1,2 & 100 \\
\hline $\mathrm{m} 489$ & 100 & $0.1-10$ & 5 & 100 \\
\hline $\mathrm{m} 491$ & 100 & $0.1-2$ & 1.08 & 100 \\
\hline $\mathrm{m} 494$ & 100 & $0.1-2$ & 1.10 & 100 \\
\hline $\mathrm{m} 495$ & 100 & $0.1-2$ & 0.5 & 100 \\
\hline m520 & 100 & $0.1-4$ & 1.0 & 100 \\
\hline m521 & 100 & $0.1-0.7$ & 0.945 & 100 \\
\hline m522 & 100 & $0.1-0.75$ & 0.95 & 100 \\
\hline m523 & 100 & $0.1-2$ & 0.94 & 50 \\
\hline m526 & 100 & $0.1-2$ & 1.0 & 10 \\
\hline p062 & 100 & $0.1-2$ & 10.0 & 50 \\
\hline p063 & 100 & $0.1-2$ & 10.0 & 50 \\
\hline p064 & 100 & $0.1-2$ & 1.0 & 50 \\
\hline p065 & 100 & $0.1-2$ & 1.0 & 50 \\
\hline p066 & 100 & $0.1-2$ & 1.0 & 50 \\
\hline p067 & 50 & $0.1-2$ & 0.5 & 50 \\
\hline p068 & 100 & $0.1-2$ & 1.0 & 50 \\
\hline p069 & 25 & $0.1-2$ & 0.25 & 50 \\
\hline p070 & 100 & $0.1-2$ & 1.0 & 100 \\
\hline p071 & 100 & $0.1-20$ & 1.0 & 10 \\
\hline p072 & 100 & $0.1-20$ & 1.0 & 10 \\
\hline p081 & 100 & $0.1-20$ & 0.25 & 10 \\
\hline p082 & 100 & $0.1-20$ & 0.1 & 10 \\
\hline p083 & 100 & $0.1-4$ & 1.0 & 100 \\
\hline p084 & 100 & $0.1-0.5$ & 1.0 & 1000 \\
\hline p085 & 100 & $0.1-10$ & 1.0 & 50 \\
\hline p086 & 100 & $0.1-20$ & 2.0 & 10 \\
\hline p087 & 100 & $0.1-20$ & 0.5 & 10 \\
\hline p088 & 100 & $0.1-200$ & 1.0 & $1.0,50$ \\
\hline p089 & 100 & $0.25-0.75$ & $0.25-2.0$ & 100 \\
\hline p090 & 100 & $0.1-20$ & 5.0 & 10 \\
\hline p091 & 100 & 2,3 & 1.0 & 10 \\
\hline p092 & 150 & $0.1-2$ & 1.5 & 50 \\
\hline
\end{tabular}

${ }^{\mathrm{a}}$ Belville disc springs were inserted into the load column for experiments p066-p092.

external signal with a constant voltage reference that was used to maintain the mean normal stress. Vibration periods $T$ ranged from 0.1 to $200 \mathrm{~s}$ and amplitudes $A$ ranged from 0.1 to $10 \mathrm{MPa}$. Experiments were conducted at room temperature $\left(22^{\circ}-25^{\circ} \mathrm{C}\right)$ and humidity $(9-33 \%)$, with the exception of $\mathrm{m} 485, \mathrm{~m} 489, \mathrm{~m} 491$, and $\mathrm{m} 494$, which were saturated with water prior to shearing. The maximum displacement, $d$, obtained during an experiment was $28 \mathrm{~mm}$, a shear strain of about 19 .

\section{Results}

[12] We evaluated the effect of normal stress vibrations by comparing to periods of constant normal stress (Figures 1 and 2). Vibration period had the greatest effect of all tested parameters, including vibration amplitude, loading rate, and mean normal stress. At short periods $(T=0.1-0.75 \mathrm{~s})$ the effect of vibrations was negligible. Intermediate-period vibrations $(T=1.0-5.0 \mathrm{~s})$ weakened the shear zone, producing an irregular (often sawtooth-shaped) response that phase lagged the $\sigma_{n}$ vibrations. At long periods $(T \geq 5 \mathrm{~s})$ the frictional response was sinusoidal and in phase, such that the coefficient of friction remained approximately constant. Normal stress vibrations always began with an increase in $\sigma_{n}$ and the first peak in $\tau$ was often larger than subsequent strength oscillations (Figure 2).
[13] Figure 3 shows the technique used to quantify observed frictional response for two cases with different amplitude vibrations $\left(\epsilon=A \sigma_{n}=0.01\right.$ and 0.05$)$. We measured three quantities for each vibration interval: (1) the steady state frictional strength variation during vibrations, $\Delta \tau$; (2) the change in peak yield strength, $\Delta \tau_{\text {yield }}$, which is the difference between the shear strength prior to vibrations, $\tau_{s s}$, and the steady state peak strength during vibrations, $\tau_{\text {peak }}$; and (3) the phase delay, $\Delta \phi$, which is defined as the time difference between peaks in the $\sigma_{n}$ and $\tau$ oscillations normalized by the vibration period. Figures 4-6 illustrate the variation in $\Delta \tau, \Delta \tau_{\text {yield }}$, and $\Delta \phi$
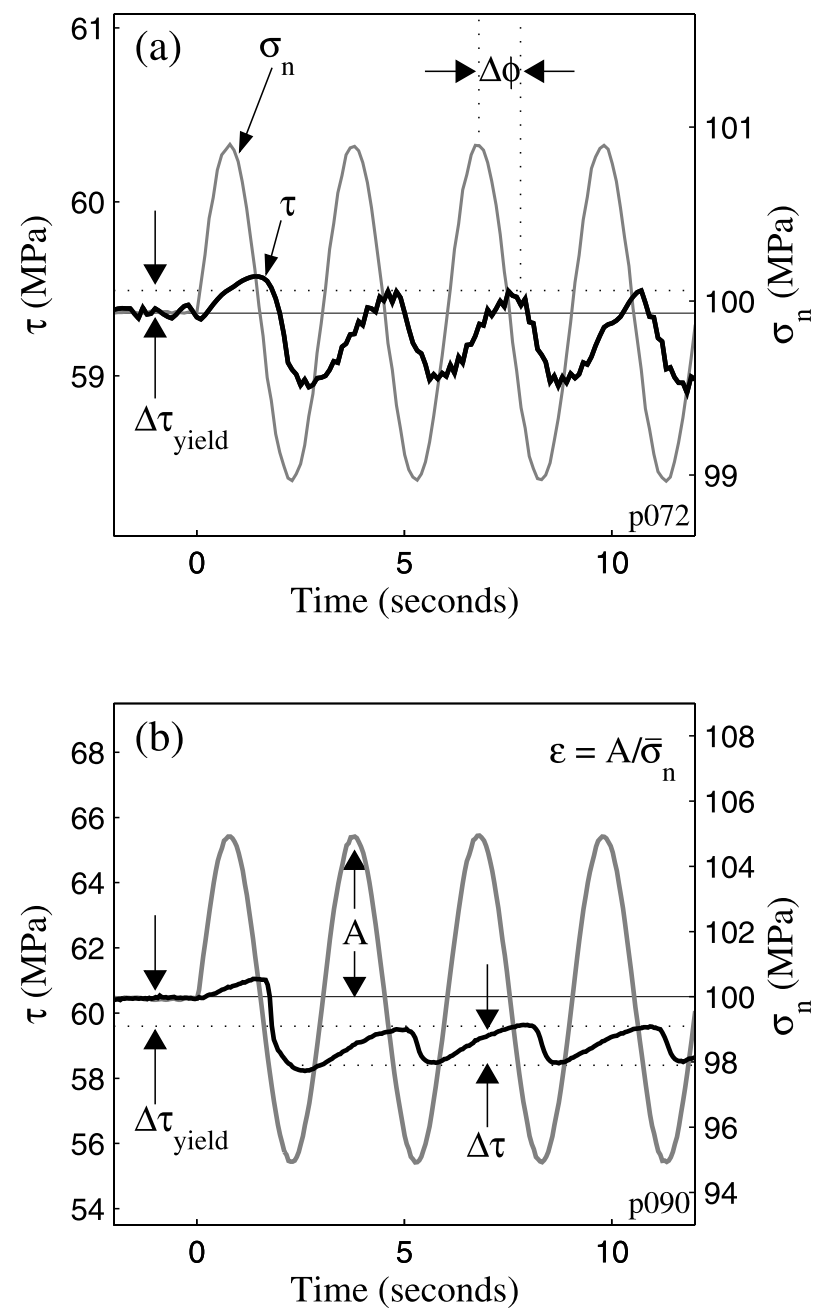

Figure 3. Quantification of the friction response to $\sigma_{n}$ vibrations. (a) $T=5 \mathrm{~s}$ and $A=1 \mathrm{MPa}$. (b) $T=5 \mathrm{~s}$ and $A=$ $5 \mathrm{MPa}$. The change in peak yield strength $\left(\Delta \tau_{\text {yield }}\right)$ is the difference between the steady state peak $\tau$ and the initial level prior to vibrations. Note that $\Delta \tau_{\text {yield }}$ can be positive or negative, indicating induced strengthening (Figure $3 \mathrm{a}$ ) or weakening (Figure $3 \mathrm{~b}$ ), respectively. $\Delta \tau$ is the peak-to-peak amplitude frictional strength response to $\sigma_{n}$ vibrations. Phase lag $(\Delta \phi)$ is the time difference between the peaks of the $\sigma_{n}$ and $\tau$ oscillations converted to angular units. Figures $3 \mathrm{a}$ and $3 \mathrm{~b}$ show 1.0 and $1.65 \mathrm{~s}$ time shifts, corresponding to $\Delta \phi=(2 / 5) \pi$ and $(2 / 3) \pi$, respectively. 
with normalized amplitude $\epsilon$, loading rate $V$, mean normal stress $\bar{\sigma}_{n}$, and vibration period $T$.

\subsection{Reproducibility and Noise}

[14] Experimental noise, such as small stick-slip events caused by grain breakage, can obscure systematic frictional strength variation. To minimize these effects we removed noise from our data before reporting $\Delta \tau$ and $\Delta \tau_{\text {yield }}$ measurements. The full amplitude of the noise was typically between $0.05-0.5 \mathrm{MPa}$, with a median of $0.25 \mathrm{MPa}$ (Figure 2). We measured the noise level prior to each vibration set and subtracted it from the full range of yield strength values to get $\Delta \tau$. Background stick-slip events have little affect on peak strength levels, thus we subtracted half of the measured noise level to obtain $\Delta \tau_{\text {yield }}$.

[15] The uncertainty in determining $\Delta \tau$ was tested using a nonlinear least squares technique. For each experiment we fit the sinusoidal function $\tau=\hat{A} \sin (2 \pi$ $(t+\Delta \hat{\phi}) / T)$ to an observed time series of $\tau$. We chose long-period time series because of their sinusoidal shape. Estimates of $\hat{A}$ and $\Delta \hat{\phi}$ and the corresponding $95 \%$ confidence intervals were obtained from a nonlinear least squares regression. The amplitude estimate $\hat{A}$ was then transformed into a noise estimate by subtracting $\hat{A}$ from the measured peak-to-peak amplitude of the shear stress oscillations, i.e., the peak-to-trough distance including excursions due to noise. The $95 \%$ confidence interval included our measured noise values for the long-period vibrations. This simple test could not be successfully applied to shorter-period vibrations, because a sine wave is not a good approximation to the shape of the frictional response. The good agreement between the estimated and measured values of $\tau$ at long periods indicates that our method of removing experimental noise is effective.

[16] Bars at the lower right of each panel of Figures 46 show the span of experimental results obtained for vibration sets conducted with the range of slip rates, normal stresses, and vibration periods listed in Table 1 . These bars represent the experimental reproducibility, and thus are considered the experimental uncertainty for our data.

\subsection{Effect of Vibration Period, $T$}

\subsubsection{Variation of Frictional Strength, $\Delta \tau^{\prime}$}

[17] A normalized frictional strength variation of $\Delta \tau^{\prime}=$ $\Delta \tau /\left(2 A \mu_{s s}\right)=1$ represents the expected $\tau$ response to $\sigma_{n}$ vibrations, assuming a constant coefficient of friction. Figures $4 \mathrm{a}, 5 \mathrm{a}$, and $6 \mathrm{a}$ show that for all tested vibration amplitudes, loading rates, and normal stresses, the frictional response was not able to "keep up" with short-period vibrations, resulting in values of $\Delta \tau^{\prime}$ near zero. In contrast, the coefficient of friction remained nearly constant for longperiod vibrations, i.e., $\Delta \tau^{\prime}$ approached 1.0.

\subsubsection{Dynamic Weakening/Strengthening, $\Delta \tau_{\text {yield }}^{\prime}$}

[18] Figures $4 \mathrm{~b}, 5 \mathrm{~b}$, and $6 \mathrm{~b}$ show the effect of $T$ on the degree of dynamic weakening or strengthening, i.e., the normalized change in peak yield strength, $\Delta \tau_{\text {yield }}^{\prime}=\Delta \tau_{\text {yield }} /$ $\left(A \mu_{s s}\right)$. Short-period vibrations either did not affect the peak strength or caused dynamic weakening $\left(\Delta \tau_{\text {yield }}^{\prime} \leq 0\right)$. In contrast, long-period vibrations always increased the peak

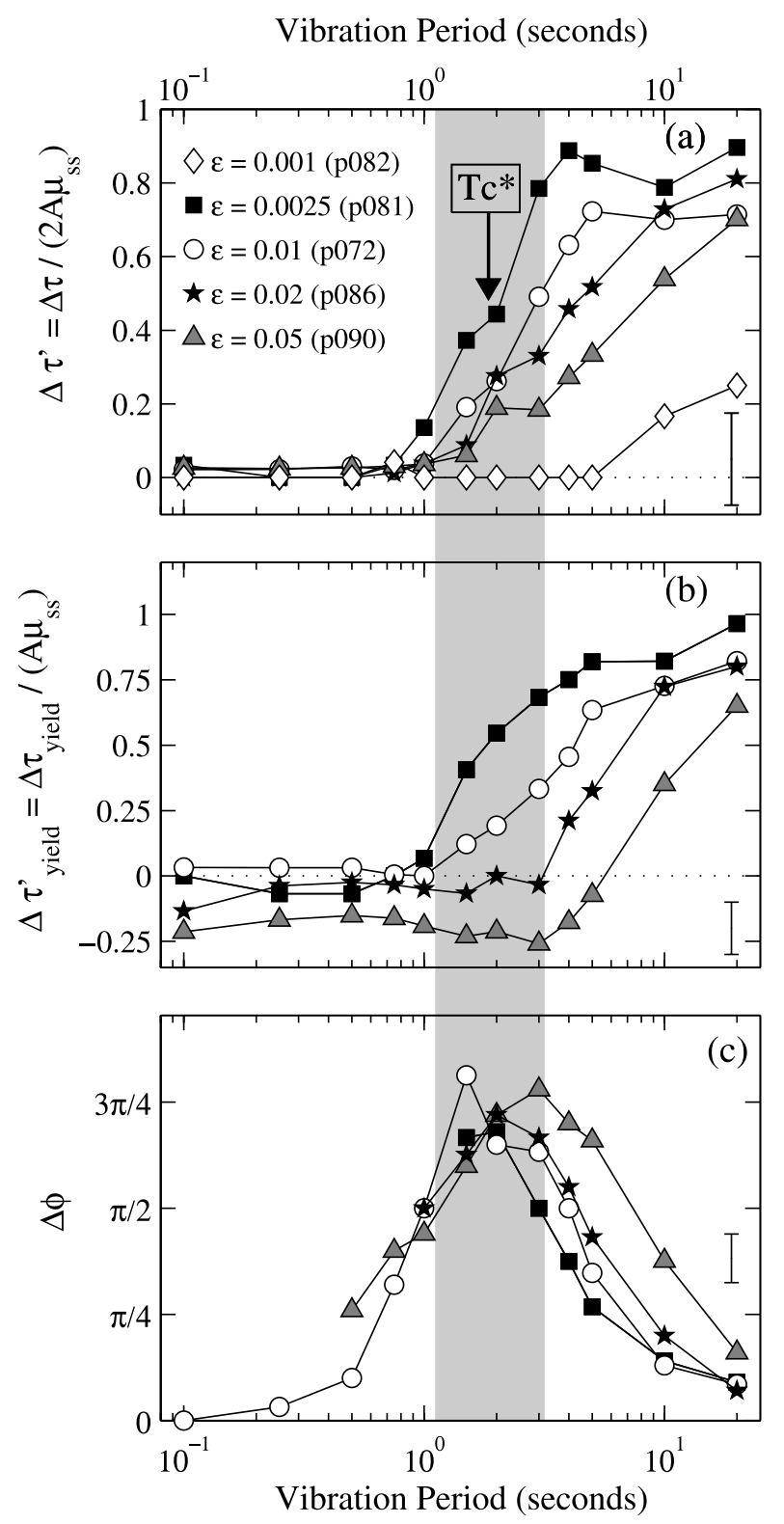

Figure 4. Effect of vibration amplitude and period on frictional strength. (a) A critical period ( $\left.T_{c}^{*}\right)$, defined at $\Delta \tau^{\prime}=$ 0.2 (shaded bar), increased with normalized vibration amplitude $(\epsilon)$. Near $T_{c}^{*}$, frictional strength transitioned from a constant level $\left(\Delta \tau^{\prime}=0\right)$ to having large-amplitude oscillations $\left(\Delta \tau^{\prime}=1\right)$. (b) Note that dynamic weakening $\left(\Delta \tau_{\text {yield }}^{\prime}<0\right)$ resulted from large-amplitude, short-period vibrations. (c) The largest phase lags $(\Delta \phi)$ were observed near $T_{c}^{*}$, and in-phase responses were seen at both long and short periods. For some vibration sets, $\Delta \phi$ was incoherent and could not be measured. The bars in the lower right of each panel (here and in figures to follow) show experimental reproducibility. Note that $\epsilon=0.001$ data are at the limit of our measurement resolution and are only plotted in Figure 4a. All data are from experiments run at $V=10 \mu \mathrm{m} / \mathrm{s}$ and $\bar{\sigma}_{n}=100 \mathrm{MPa}$, with $d=6.5-10 \mathrm{~mm}$ (strains of $4.3-6.7)$. 

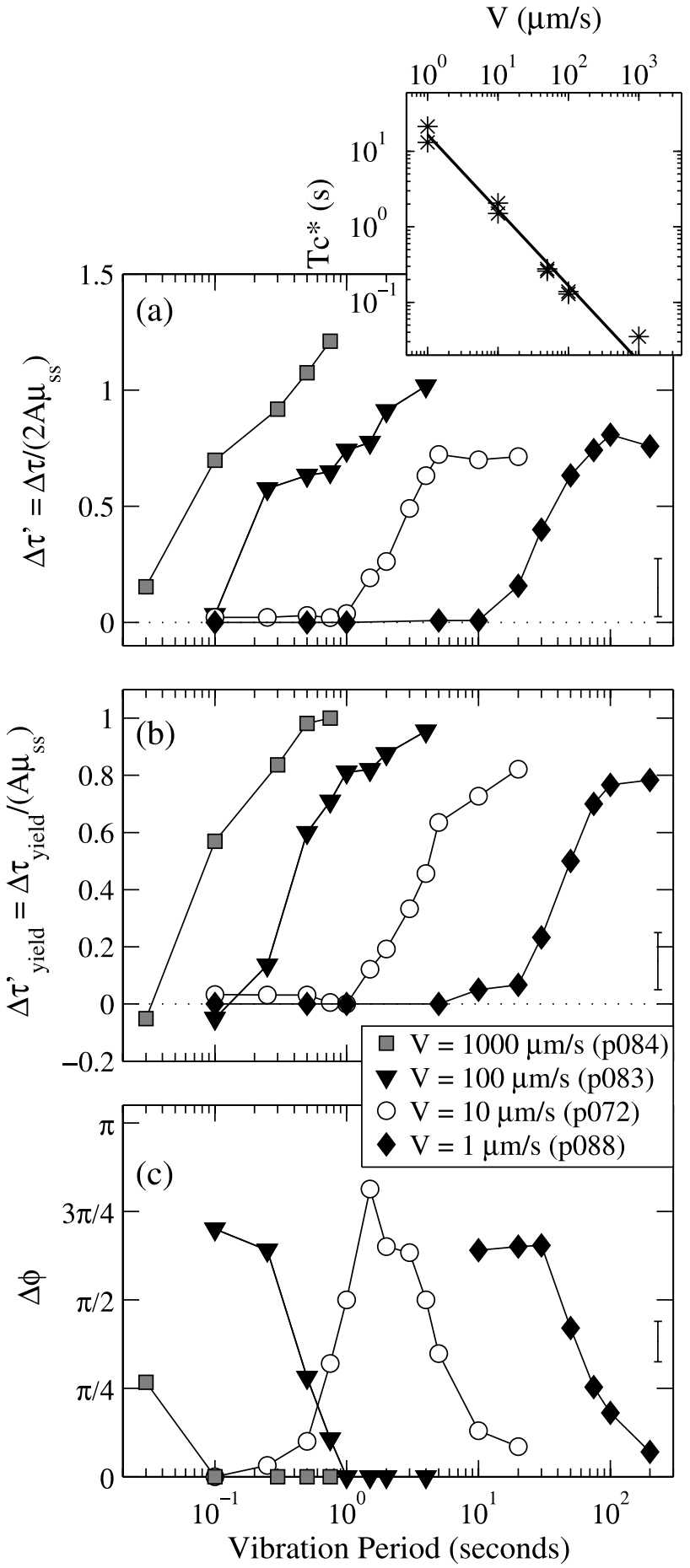

Figure 5. Effect of loading rate and vibration period on frictional strength. $\Delta \tau^{\prime}, \Delta \tau_{\text {yield }}^{\prime}$, and $\Delta \phi$ behaved systematically for all loading rates: Near $T_{c}^{*}$, strength oscillations increased in amplitude and phase lag reached a maximum. $T_{c}^{*}$ decreased linearly with $V$, as shown in the inset. (a) Note that $\Delta \tau^{\prime}>1$ was observed for long-period tests with $V \geq$ $100 \mu \mathrm{m} / \mathrm{s}$. (b) Also note that only the fastest runs showed dynamic weakening $\left(\Delta \tau_{\text {yield }}^{\prime}<0\right)$. (c) Only the $V=10 \mu \mathrm{m} / \mathrm{s}$ test showed coherent short-period $\Delta \phi$ data. All experiments were conducted with $\bar{\sigma}_{n}=100 \mathrm{MPa}, \epsilon=0.01$, and $d=6-$ $20 \mathrm{~mm}$ (strains of $4-13.5$ ). yield strength $\left(\Delta \tau_{\text {yield }}^{\prime}>0\right)$. At the longest periods $\tau$ oscillated around $\tau_{s s}$ without causing an alteration in the time-averaged strength of the shear zone.

\subsubsection{Phase Lag, $\Delta \phi$}

[19] Phase lag $\Delta \phi$, shown in Figures $4 \mathrm{c}, 5 \mathrm{c}$, and 6c, corresponds to a time or displacement scale needed to change frictional strength. When $\Delta \phi=0$, the time or slip needed to effect a change in friction is short compared to the vibration period for a given loading rate. We find a peak in $\Delta \phi$ near the minimum $\Delta \tau_{\text {yield }}^{\prime}$. At short periods, $\Delta \phi$ could not always be obtained because either $\tau$ oscillated with a period that was a multiple or fraction of $\sigma_{n}$, or no systematic $\Delta \phi$ was observable. The few short-period oscillations for which $\Delta \phi$ was measured suggest a tendency toward an in-phase response at short periods.

3.2.4. Critical Period, $T_{c}^{*}$

[20] A transition in $\Delta \tau^{\prime}, \Delta \tau_{\text {yield }}^{\prime}$, and $\Delta \phi$ occurs at a critical period $T_{c}^{*}$ dependent on experimental parameters (shaded bar in Figure 4). We define $T_{c}^{*}$ as the period at which $\Delta \tau^{\prime}=0.2$. This corresponds to the minimum $\Delta \tau_{\text {yield }}^{\prime}$ and the maximum $\Delta \phi . T_{c}^{*}$ is minimally affected by vibration amplitude and normal stress, but varies systematically with loading rate (see inset of Figure 5).

\subsection{Effect of Epsilon, $\epsilon$}

[21] We studied the effect of vibration amplitudes $\epsilon=$ $A / \bar{\sigma}_{n}$ between 0.001 and 0.05 (Figure 4). The total shear strength variation, $\Delta \tau$, increased linearly with $\epsilon$ (compare amplitudes shown in Figure 3). In Figure 4 we plot normalized quantities, which remove the first-order effect of amplitude. The normalization enables direct comparison of the $\epsilon$ tests at the same scale. $T_{c}^{*}$ was near $2 \mathrm{~s}$ for all $\epsilon$, with a slight, systematic increase in $T_{c}^{*}$ with $\epsilon$. Short-period, large-amplitude vibrations significantly affected $\Delta \tau_{\text {yield }}^{\prime}$, producing the weakening shown in Figure $4 \mathrm{~b}$. All experiments shown in Figure 4 were loaded at $V=10 \mu \mathrm{m} / \mathrm{s}$ under a mean normal stress of $\bar{\sigma}_{n}=100 \mathrm{MPa}$. The effect of very small $\epsilon$ vibrations $(<0.0025)$ was below our detection limit (e.g., $\epsilon=0.001$ in Figure 4a). Therefore only runs with $\epsilon \geq 0.0025$ were included in our analysis and in Figures $4 \mathrm{~b}$ and $4 \mathrm{c}$. Owing to the normalization of $\Delta \tau^{\prime}$ and $\Delta \tau_{\text {yield }}^{\prime}$ by $\epsilon$, the two smallest-amplitude experiments shown in Figure 4 $(\epsilon<0.01)$ have larger uncertainties than those shown with the bars, and the large-amplitude tests $(\epsilon>0.01)$ have smaller uncertainties.

\subsection{Effect of Sliding Rate, $V$}

[22] We studied the effect of loading rate $V$ between $1-1000 \mu \mathrm{m} / \mathrm{s}$ and found that $T_{c}^{*}$ decreases systematically with $V$ (Figure 5). More subtle effects of $V$ on $\Delta \tau^{\prime}$ and $\Delta \tau_{\text {yield }}^{\prime}$ were also observed. Long-period vibrations at the faster loading rates $(V=100,1000 \mu \mathrm{m} / \mathrm{s})$ resulted in $\Delta \tau^{\prime}>1$ and $\Delta \tau_{\text {yield }}^{\prime} \approx 1.0$ and short periods caused slightly negative $\Delta \tau_{\text {yield }}^{\prime}$ values, suggestive of dynamic weakening. At the slower loading rates $(V=1,10 \mu \mathrm{m} / \mathrm{s})$, neither $\Delta \tau^{\prime}$ nor $\Delta \tau_{\text {yield }}^{\prime}$ reached 1.0 , indicating that the coefficient of friction varied even during long-period vibrations. The inset in Figure 5 displays the linear variation in $T_{c}^{*}$ with $V$. Measurements are from the four experiments shown in Figures $5 \mathrm{a}-5 \mathrm{c}$ as well as additional 
vibration tests at the same conditions. Performing a least squares regression, we found $T_{c}^{*}=16.6 / \mathrm{V}$.

\subsection{Effect of Normal Stress, $\bar{\sigma}_{\boldsymbol{n}}$}

[23] We explored the effect of the stiffness ratio $k / k_{c}$ by conducting experiments with and without springs in the load column (Table 1) and using mean normal stress of $\bar{\sigma}_{n}=$ 50,100 , and $150 \mathrm{MPa}$ (Figure 6). The springs reduce $k$, the
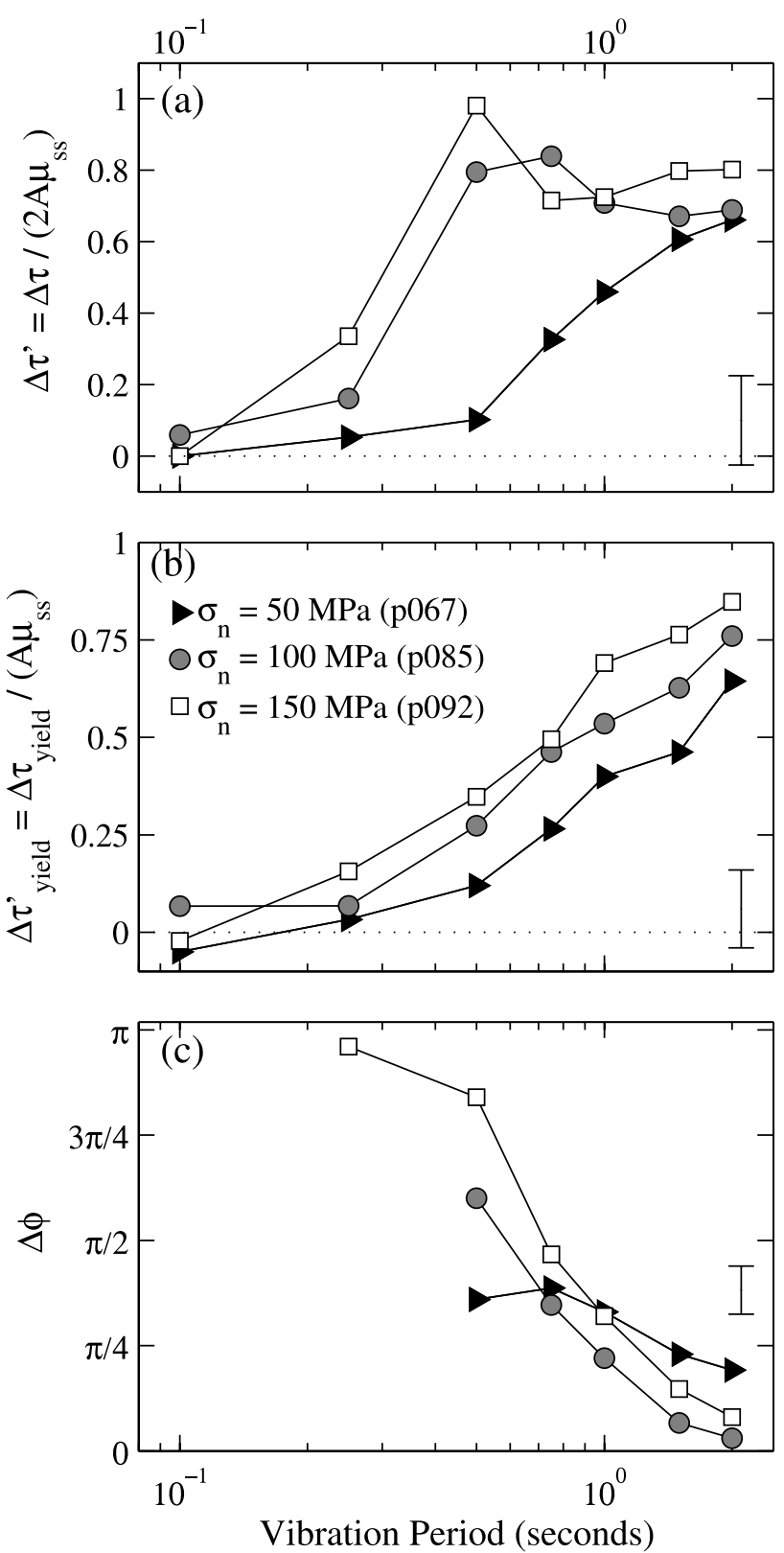

Figure 6. Effect of normal load and vibration period on frictional strength. The period at which $\Delta \tau^{\prime}=0.2\left(T_{c}^{*}\right)$ decreased slightly with $\bar{\sigma}_{n}$. (a) Note the peak in $\Delta \tau^{\prime}$ at $T=$ $0.5 \mathrm{~s}$ for $\bar{\sigma}_{n}=150 \mathrm{MPa}$. (b) The relative amplitude of $\Delta \tau_{\text {yield }}^{\prime}$ for the three tests shows the decrease in $T_{c}^{*}$ with $\bar{\sigma}_{n}$. (c) At the highest $\bar{\sigma}_{n}$ the shear stress response was fully out of phase with the $\sigma_{n}$ vibrations. All experiments were conducted with $V=50 \mu \mathrm{m} / \mathrm{s}, \epsilon=0.01$, and $d=7-14 \mathrm{~mm}$ (strains of 4.7-9.3).
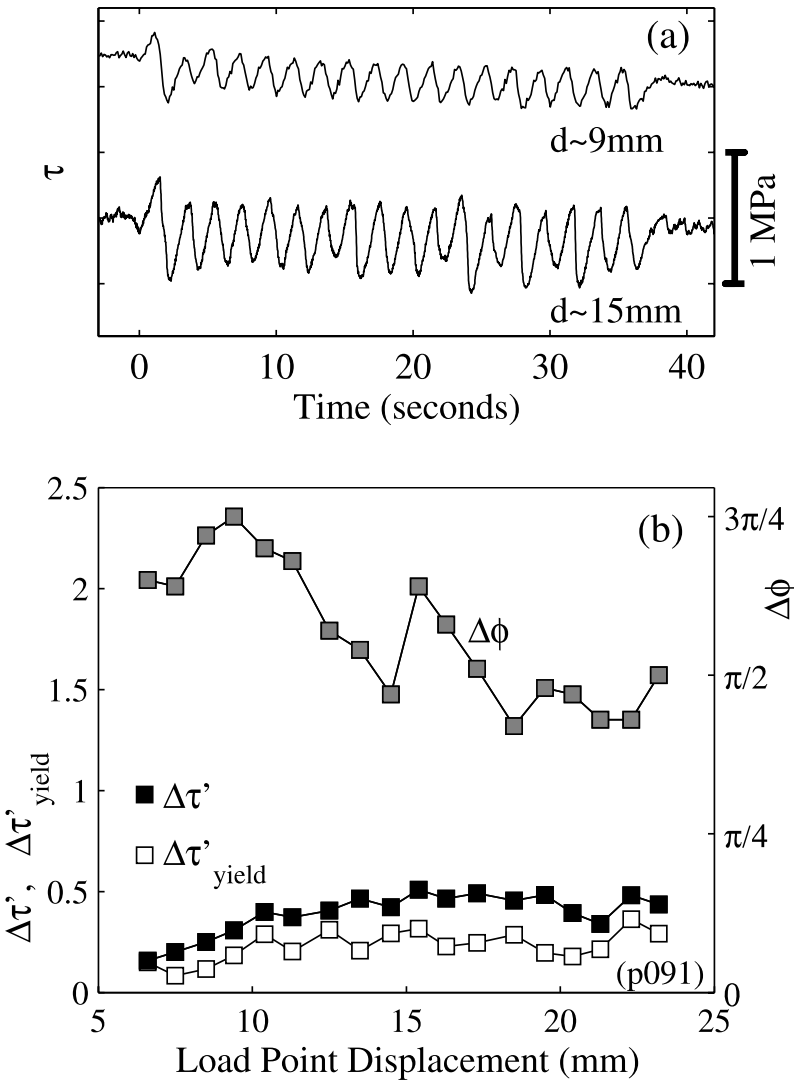

Figure 7. Effect of net shear strain on frictional strength. (a) Time series from $d=9 \mathrm{~mm}$ and $d=15 \mathrm{~mm}$ vary slightly in amplitude of both the frictional response and experimental noise. (b) $\Delta \tau^{\prime}, \Delta \tau_{\text {yield }}^{\prime}$, and $\Delta \phi$ are plotted as a function of load point displacement (e.g., Figures 4-6). Note that both $\Delta \tau^{\prime}$ and $\Delta \tau_{\text {yield }}^{\prime}$ changed very little after $d \approx$ $10 \mathrm{~mm}$ (shear strain of about 6.6). Phase lag was the least stable quantity, but it remained high (near $\pi / 2$ ) for all displacements. All data are from a single experiment with parameters $\bar{\sigma}_{n}=100 \mathrm{MPa}, \epsilon=0.01, V=10 \mu \mathrm{m} / \mathrm{s}$, and $T=2 \mathrm{~s}$.

stiffness of our testing apparatus, by approximately an order of magnitude, and $k$ increases with $\sigma_{n}$ (as described in the experimental procedure). $k_{c}$, the critical stiffness, is given by

$$
k_{c}=\frac{\sigma_{n}(b-a)}{D_{c}}\left(1+\frac{m V^{2}}{a \sigma_{n} D_{c}}\right),
$$

[Rice and Ruina, 1983], where $a, b$, and $D_{c}$ are empirical friction parameters (and are discussed further below), and $m$ is the mass of the system. A change in $\bar{\sigma}_{n}$ produces a greater effect on $k_{c}$ than on $k$. Thus an increase in $\bar{\sigma}_{n}$ decreases $k / k_{c}$, bringing the system closer to the critical state, $k=k_{c}$.

[24] Figure 6 shows that the highest-normal stress (lowest $k / k_{c}$ ) resulted in the least systematic frictional behavior. The response to $T=0.5 \mathrm{~s}$ vibrations with $\bar{\sigma}_{n}=150 \mathrm{MPa}$ is suggestive of the resonance seen in the numerical simulations of Perfettini et al. [2001]. The frictional strength oscillations, at vibration periods near $T_{c}^{*}$ and the lowest $k / k_{c}$, were fully out of phase with $\sigma_{n}$. Both the large phase lags and the amplified shear strength response, indicate that 
the system was closer to a critical state during low $k / k_{c}$ experiments. The use of the Belleville disc springs and elevated $\sigma_{n}$ conditions both helped to reduce the difference between the actual system stiffness and the theoretical critical stiffness, however our apparatus remained intrinsically too stiff to observe strong resonant behavior.

\subsection{Effect of Displacement, $d$}

[25] One limitation of these experiments was the small range of displacement available for study. In larger-displacement experiments [e.g., Beeler et al., 1994; Mair and Marone, 1999] significant evolution of the shear zone was observed during initial slip, depending on $\sigma_{n}$. We attempted to minimize the displacement needed to obtain a steady state shear zone fabric by imposing load cycles and velocity steps at the start of each run. While the preconditioning helped, our shear zones continued to evolve at a reduced rate throughout the experiment.

[26] Data obtained from vibration sets at equal displacements were compared whenever possible. In Figures 4 and 6 , where $V=10$ and $50 \mu \mathrm{m} / \mathrm{s}$, respectively, we have displayed results from the first set of $\sigma_{n}$ vibrations. However, in order to compare experiments conducted at different loading rates (Figure 5), it was necessary to use measurements from the entire range of displacement. The second and third vibration sets (see Figure $1, d>11 \mathrm{~mm}$ ) show the same systematics as the first set, however the increased frequency and amplitude of stick-slip events, which occur both during vibrations and under constant normal load, made obtaining precise measurements more difficult.

[27] In Figure 7a we show two shear stress time series taken from a single experiment with $V=10 \mu \mathrm{m} / \mathrm{s}, \epsilon=0.01$, $\sigma_{n}=100 \mathrm{MPa}$, and $T=2 \mathrm{~s}$. The first is from $9 \mathrm{~mm}$ of displacement, and the second, with large-amplitude oscillations and an elevated noise level, is from $15 \mathrm{~mm}$ of displacement. Figure $7 \mathrm{~b}$ illustrates measurements from the entire range of obtainable displacements. Very little change occurs in either $\Delta \tau^{\prime}$ or $\Delta \tau_{\text {yield }}^{\prime}$ past $d=9 \mathrm{~mm}$. The $\Delta \phi$ measurements are less stable than those of shear strength. The period shown in Figure 7, $T=2 \mathrm{~s}$, is near $T_{c}^{*}$ for the displacement rate $V=10 \mu \mathrm{m} / \mathrm{s}$ (see Figure $5 \mathrm{~d}$ ). Phase lag measurements in all experiments are observed to fluctuate most strongly near $T_{c}^{*}$. The variation in $\Delta \phi$ shown in Figure $7 \mathrm{~b}$ is not large when compared with variation in $\Delta \phi$ observed for different vibration periods. Thus although displacement does have an effect on the values we report, it is minimal when compared to the effects of $T, V$, and $\epsilon$.

\subsection{Effect of Humidity}

[28] Water content is known to affect frictional behavior [Frye and Marone, 2002]. We conducted four experiments under saturated conditions with $V=100 \mu \mathrm{m} / \mathrm{s}$ and $\bar{\sigma}_{n}=$ $100 \mathrm{MPa}$. Stable sliding at constant frictional strength was observed during short-period vibrations and sinusoidal oscillations resulted from long periods. Between the simple behaviors observed at short and long periods, the response at intermediate periods $(0.25 \leq T \leq 0.75 \mathrm{~s})$ was complex. Large drops in frictional strength, which approached a magnitude equal to $A$, occurred regularly with periods both above and below the vibration period. These large-amplitude instabilities are consistent with a more evolved shear zone and intrinsically more unstable frictional behavior.

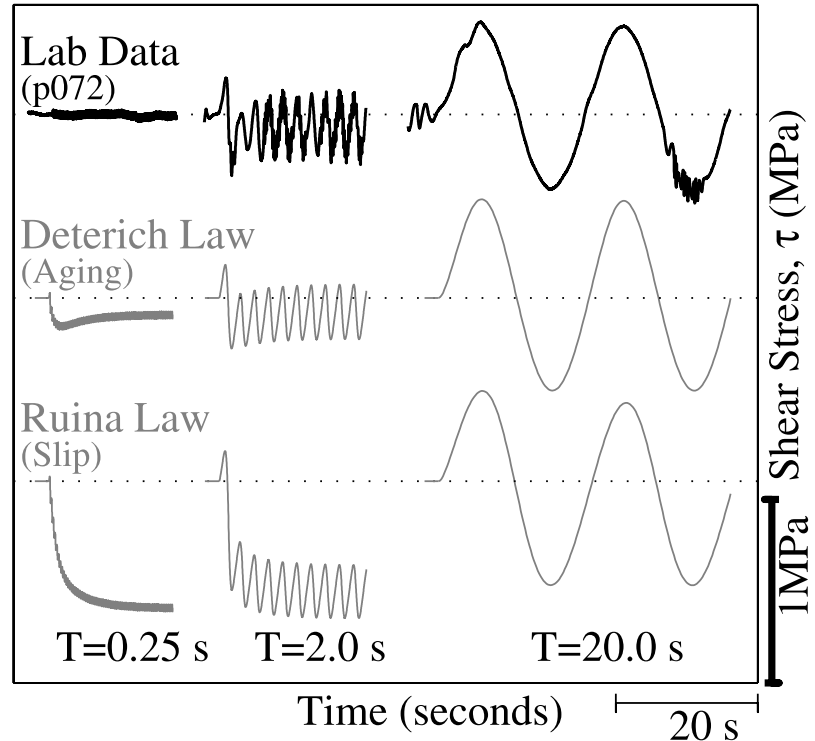

Figure 8. Time series of frictional strength for both laboratory data (black line) and Dieterich and Ruina rate/ state friction models (gray lines). Note the discrepancy at $T=0.25 \mathrm{~s}$ between the predicted weakening in the models (greatest for the Ruina law) and the constant strength observed in the experimental data. The Ruina law also predicted weakening at $T=2.0 \mathrm{~s}$. The parameters used in both models (as well as the simulations shown in the following figures) are $V=10 \mu \mathrm{m} / \mathrm{s}, \epsilon=0.01, \bar{\sigma}_{n}=100 \mathrm{MPa}$, $k / k_{c}=4, a=0.0015, b=0.0065, D_{c}=60 \mu \mathrm{m}$, and $\alpha=0.3$. The laboratory experiment was run at $V=10 \mu \mathrm{m} / \mathrm{s}, \epsilon=0.01$, and $\bar{\sigma}_{n}=100 \mathrm{MPa}$.

Although much can be learned from experiments in which the sample is near a critical stability transition, for this initial study we chose to focus on the systematic behavior of the more stable, low-water-content conditions.

\section{Discussion}

[29] The effects of normal stress vibrations on creeping shear zones are systematic and can be modeled through numerical simulations with a single set of friction parameters. We use rate and state constitutive friction laws of the standard form [e.g., Ruina, 1983; Marone, 1998]:

$$
\mu=\mu_{0}+a \ln \left(\frac{V}{V_{0}}\right)+b \ln \left(\frac{V_{0} \theta}{D_{c}}\right)
$$

where $\mu_{0}$ is the coefficient of friction during steady sliding at velocity $V_{0}, V$ is the slip rate, $\theta$ is a state variable that evolves with either time or slip, $a$ and $b$ are empirical constants that respectively correspond to a direct effect and the state evolution following a change of velocity or normal stress, and $D_{c}$ is the critical slip distance needed for $\mu$ to evolve to a new steady state following a change in loading conditions. In the following numerical simulations (Figures 8-15), we used values of $a=0.0015, b=0.0065$, and $D_{c}=60 \mu \mathrm{m}$, which were chosen from the range of behaviors found from modeled velocity steps in our experiments. We 
compare laboratory data directly to our model results and discuss the numerical simulations of Perfettini et al. [2001], whose work predicts much of the systematic behavior we observe.

\subsection{Evolution of the State Variable}

[30] The two most often cited formulations for the friction state evolution are indistinguishable when linearized around steady state, but predict different behavior when subject to normal stress perturbations [Linker and Dieterich, 1992; Richardson and Marone, 1999; Bureau et al., 2000; Perfettini et al., 2001]. The Dieterich law,

$$
\frac{d \theta}{d t}=1-\frac{V \theta}{D_{c}}-\alpha \frac{\theta \dot{\sigma}}{b \sigma},
$$

in which the state evolves with time as well as slip [Dieterich, 1979; Ruina, 1983], predicts stable behavior for all cases of $k>k_{c}$, while the Ruina law,

$$
\frac{d \theta}{d t}=\frac{-V \theta}{D_{c}} \ln \left(\frac{V \theta}{D_{c}}\right)-\alpha \frac{\theta \dot{\sigma}}{b \sigma}
$$

in which the state changes only with slip [Ruina, 1983], predicts conditional stability for $k>k_{c}$. The above formulations include an extension of the standard rate and state equations to include changes in normal stress [Linker and Dieterich, 1992; Richardson and Marone, 1999; Perfettini et al., 2001]. The term $\alpha$ describes the evolution of state and friction following a normal stress change from $\sigma$ to $\sigma_{0}$. Increasing $\alpha$ results in a decreased instantaneous response of the system. Linker and Dieterich [1992] observed a significant step in $\tau$ immediately following a sudden change in $\sigma_{n}$. Richardson and Marone [1999] also observed a step change in $\tau$ with a step in $\sigma_{n}$ and found $\alpha=$ 0.3 best fit their experimental data. We adopt $\alpha=0.3$ for our numerical modeling and are able to model our data reasonably well with this value (Figures $8-15$ ).

[31] We compare our experimental results to both formulations of the state evolution. Equation (3) or (4) is coupled with equation (2) and with a description of elastic interaction with our testing machine:

$$
\frac{d \mu}{d t}=k^{\prime}\left(V_{l p}-V\right)
$$

where $k^{\prime}$ is defined in terms of friction per displacement as $k^{\prime}=k /\left(\sigma_{n} A_{\text {sample }}\right), A_{\text {sample }}$ is the sample area, $V_{l p}$ is the load point velocity, and $V$ is the shearing velocity along the fault surface.

\subsection{Theory and Observations}

[32] Figure 8 shows comparisons between experimental and theoretical time series from three sets of vibrations with periods of $T=0.25,2.0,20.0 \mathrm{~s}$, loaded at $V=10 \mu \mathrm{m} / \mathrm{s}$, under a mean normal stress of $\bar{\sigma}_{n}=100 \mathrm{MPa}$. Both the Dieterich and Ruina laws fit the data well at long and intermediate periods, but neither law correctly predicts the shortest period response. Both laws overpredict the magnitude of vibration-induced weakening, however the Dieterich law is much closer to the observed values.
[33] The steady frictional strength observed experimentally for short-period vibrations (e.g., the response to $T=$ $0.25 \mathrm{~s}$ vibrations in Figure 8) implies that vibrations may not always result in an immediate step change in $\tau$, as we have assumed with $\alpha=0.3$. A higher value of $\alpha$, i.e., $\alpha=$ 0.6 , is consistent with a smooth evolution to a new steady state frictional strength, rather than an immediate change in $\tau$. However, $\alpha>0.3$ underpredicts the amplitude of the frictional response at all vibration periods and overpredicts the phase lag for long-period vibrations. Additionally, the Dieterich law with $\alpha>0.4$ predicts an overall strengthening of the shear zone with high-frequency vibrations, not the weakening that is experimentally observed.

[34] Prakash [1998] observed a continuous evolution of frictional strength following a step change in normal stress in his high-velocity $(1 \leq V \leq 30 \mathrm{~m} / \mathrm{s})$ friction experiments. His experiments, conducted on metals and without gouge, could not be described using equations (3) and (4), and instead Prakash proposed a system with two state variables. While we did not observe an immediate change in $\tau$ with all normal stress vibrations, those at large amplitudes and short periods did produce an immediate effect. We proceed here with equations (3) and (4), the formulations derived from rock friction experiments, to probe the usefulness of the Linker and Dieterich [1992] modification to the standard rate and state equations.

[35] In Figure 9 we compare laboratory measurements and predicted values of $\Delta \tau^{\prime}, \Delta \tau_{\text {yield, and }}^{\prime} \Delta \phi$. Both the Dieterich and Ruina laws provide a good fit to the $\Delta \tau^{\prime}$ data and the long-period response $\Delta \tau^{\prime}=1$ is indeed predicted when long-enough-period vibrations are imposed. The Dieterich law approximates the $\Delta \tau_{\text {yield }}^{\prime}$ measurements, but the Ruina law dramatically underpredicts the observed values. Neither the Dieterich nor the Ruina law fits the few shortperiod phase lag measurements shown in Figure 9c. Both laws predict a plateau of large phase lag at short periods, while our limited $\Delta \phi$ data suggest an in-phase response at short periods. The analytical solutions of Perfettini et al. [2001] predict the same behavior as our numerical simulations for a system with $k / k_{c}=4$ and $\alpha=\mu_{s s} / 2$. They show that at long periods, $\Delta \phi \rightarrow 0$ and at short periods, $\Delta \phi$ ramps up to $\pi / 2$. Phase lag measurements at $T<T_{c}^{*}$ are difficult to obtain and additional, well-resolved measurements are necessary to verify our short-period observations.

[36] While neither friction evolution law predicts all details of our observations, the Dieterich law provides a close fit. Additionally, the amplitude of the frictional response increases continuously with vibration amplitude, following the predictions of the Dieterich law [Perfettini et al., 2001, Figure 4]. This continuous increase can be seen when the $\Delta \tau^{\prime}$ values shown in Figure $4 \mathrm{a}$ are multiplied by the vibration amplitude of the test. Thus our data suggest that friction evolves with time as opposed to slip.

\subsection{Dynamic Weakening}

[37] Certain experimental conditions (e.g., $\mathrm{V} \geq 50 \mu \mathrm{m} / \mathrm{s}$, $\left.T<T_{c}^{*}, \epsilon>0.01\right)$ reduce the peak yield strength of the shear zone. We refer to the lowered strength as dynamic weakening, where the maximum strength reached during vibrations was reduced below the level for steady frictional sliding $\left(\Delta \tau_{\text {yield }}^{\prime}<0\right)$. This weakening effect is most pronounced with large $\epsilon$ vibrations at high frequencies 


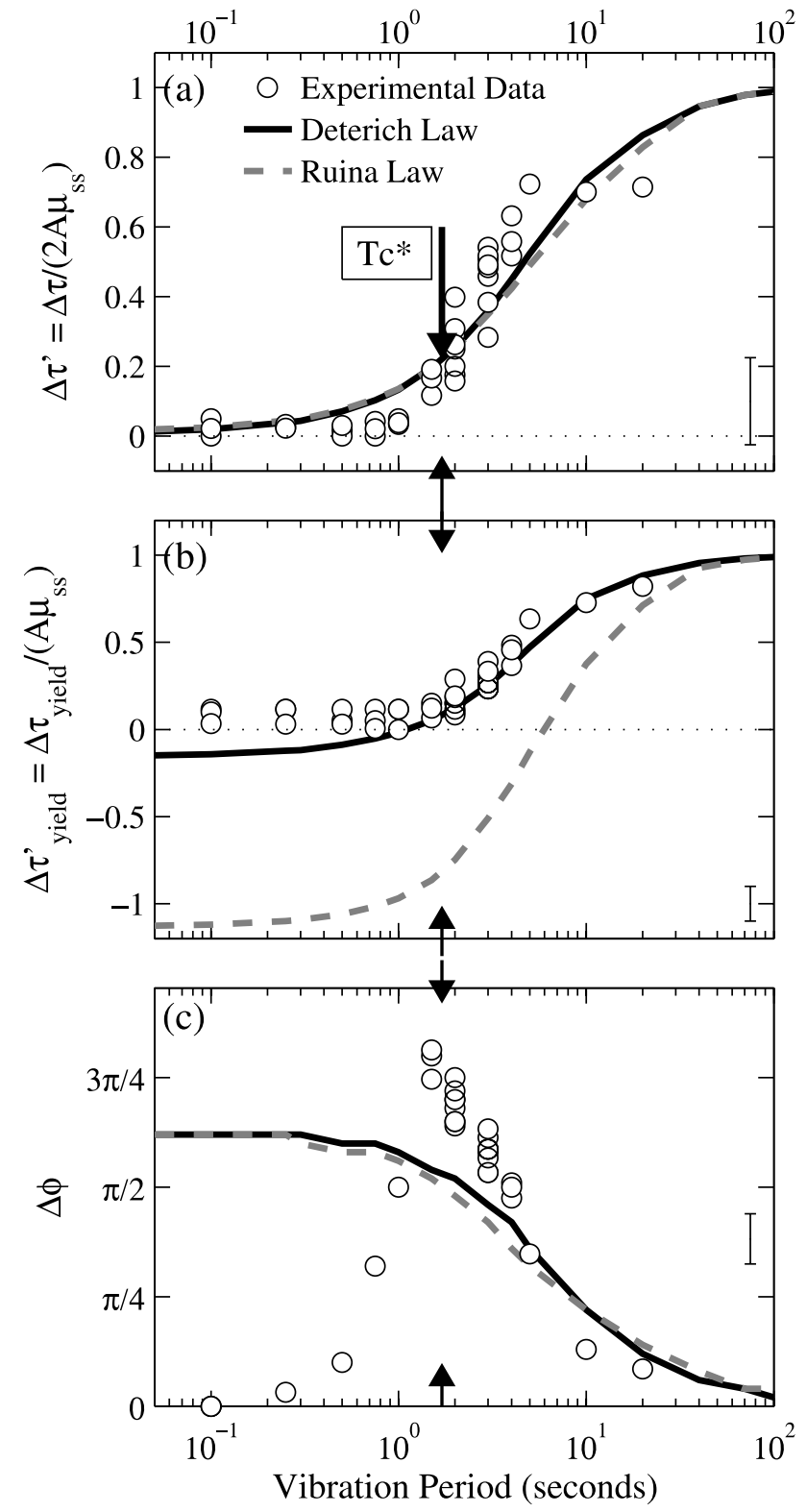

Figure 9. Effect of vibration period on frictional strength observed in laboratory data (circles) and predicted by the Dieterich (solid black line) and Ruina (dashed gray line) rate/state friction models. (a) $\Delta \tau^{\prime}$ increased with $T$ for both data and models. (b) $T_{c}^{*}$ (indicated by arrows) corresponds to the period at which $\Delta \tau_{\text {yield }}=0$ for both experimental data and the Dieterich law. The extreme weakening predicted by the Ruina law was not observed in the data. (c) Note that at short periods the data showed $\tau$ in phase with $\sigma_{n}$, while both models predicted large $\Delta \phi$. The model parameters are given in Figure 8 and the experiments were run at $V=10 \mu \mathrm{m} / \mathrm{s}$, $\bar{\sigma}_{n}=100 \mathrm{MPa}$, and $\epsilon=0.01$.

and the weakening is well modeled with the Dieterich law (Figure 10).

[38] Using a numerical model with rate-dependent and state-dependent friction, Perfettini et al. [2003] found dynamic earthquake triggering to be most effective when the faults experienced high-frequency, large-amplitude stress transients. Bureau et al. [2000] found similar results in their laboratory experiments of a sliding interface subject to high-frequency $(f=120 \mathrm{~Hz}) \sigma_{n}$ vibrations. The strength reduction in their experiments was enhanced with increased vibration amplitude, providing as much as a $20 \%$ decrease in the average coefficient of friction for $\epsilon=0.5$. The largest normal stress perturbations imposed in our experiments were an order of magnitude smaller than those of Bureau et al. [2000] and yielded a reduction in peak yield strength of about $1 \%$ (Figure 11).

[39] In our experiments, dynamic weakening was observed only when a large stress drop occurred during the initial decrease in normal stress. Figure 11 shows the effect of large-amplitude $\sigma_{n}$ vibrations on the slip rate, shear strength, and layer thickness of our sample. Close to a 45 -fold increase in shear zone slip rate occurs simultaneously with the $2.7 \mathrm{MPa}$ stress drop during the first $\sigma_{n}$ cycle. This stress drop was a $4.5 \%$ decrease in the shear zone strength, but over the full vibration set, the peak strength drop was only about $1 \%$, which corresponds to $\Delta \tau_{\text {yield }}^{\prime}=-0.24$.

[40] Four stages of frictional behavior can be observed in Figure 11. In stage I the shear zone looses strength during a fast slip event accompanied by dilation of the shear zone. Stage I may be thought of as a seismic event that resets the state of the shear zone, i.e., $\theta=\theta_{0}$. During stage II the shear zone's strength is partially recovered. Figure $11 \mathrm{c}$ shows that strength increases over three normal stress oscillations following the initial stress drop. During the recovery period, the slipping speed remains near the background loading rate and the frictional state evolves toward a more mature level. The layer compacts following the slip event (Figure 11d) and then dilates and again compacts as $\sigma_{n}$ cycles during stage II. While the vibrations continue the shear zone remains thinned compared to its original thickness under constant $\sigma_{n}$. Stage III begins where the shear strength is no longer climbing steadily and each drop in $\sigma_{n}$ induces a small slip event (at approximately $5 \mathrm{~s}$ for the example shown in

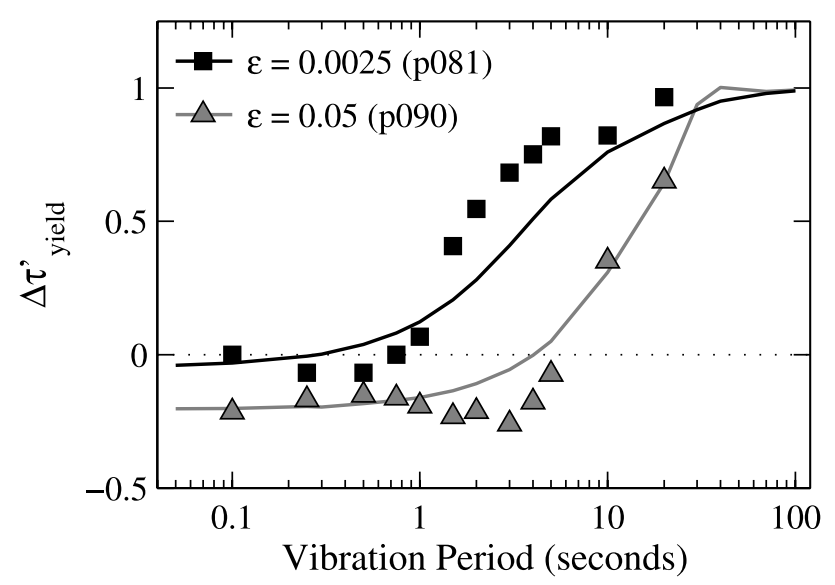

Figure 10. Effect of vibration amplitude and period on level of frictional strength observed in laboratory data (symbols) and predicted by the Dieterich rate/state friction model (lines). Large-amplitude vibrations $(\epsilon>0.01)$ caused dynamic weakening in both experimental and numerical tests. Model parameters are listed in Figure 8 with $\epsilon=$ 0.0025 and 0.05 . 

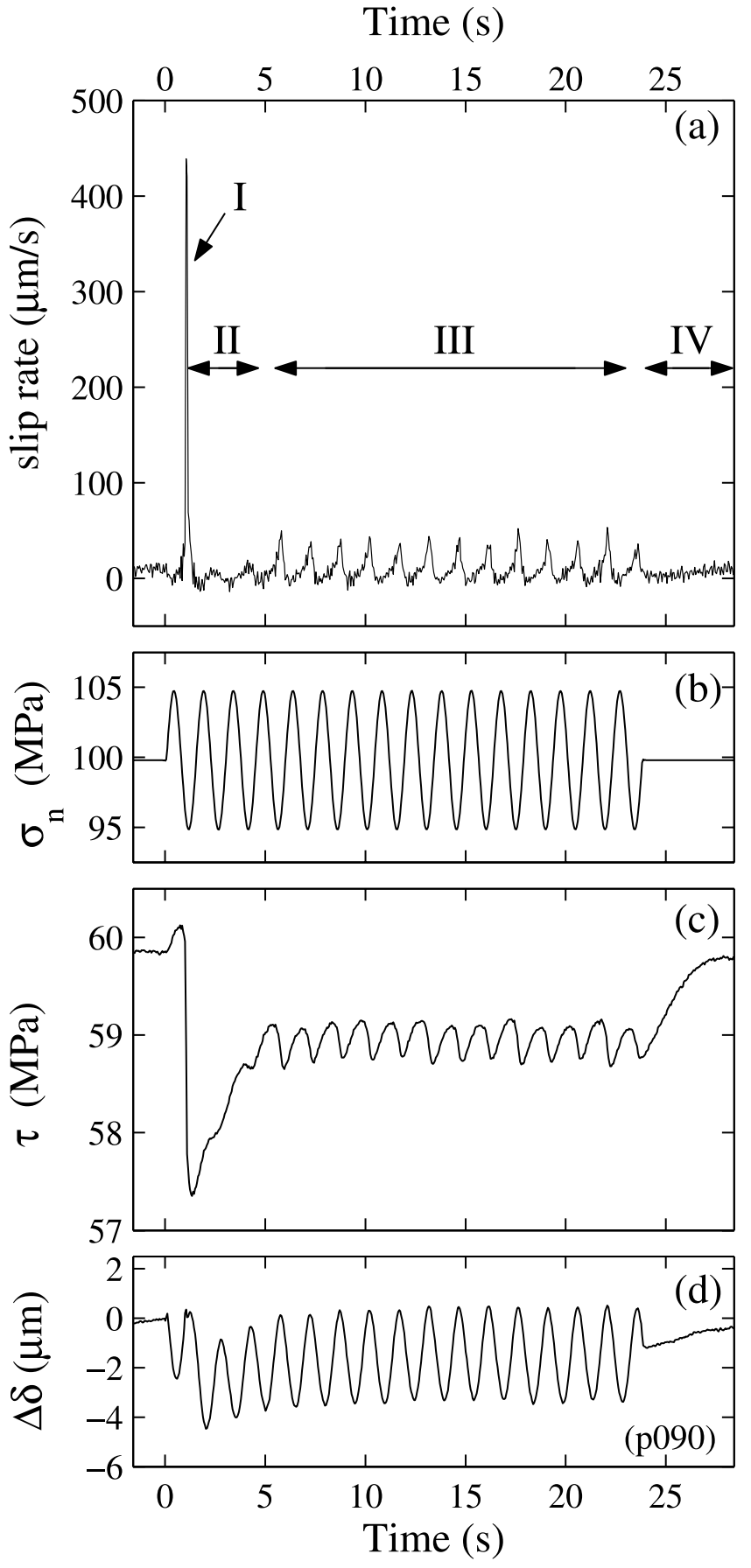

Figure 11. Time series of slip rate, normal stress, frictional strength, and layer thickness changes $(\Delta \delta)$ for large amplitude $(\epsilon=0.05)$ vibrations near $T_{c}^{*}(T=1.5 \mathrm{~s})$. Note (a) the large slip rate events, (c) the stress drops and dynamic weakening, and (d) compaction observed during vibrations. (b) Imposed vibrations started at time 0 and continued for $24 \mathrm{~s}$ through the four stages of frictional behavior described in the text.

Figure 11). We suspect that each slip event disrupts the particle packing, and thus reduces the frictional state of the system, such that the shear strength remains lower than the nonvibrated $\tau_{s s}$. The compacted layer, however, is not in steady state, but instead continues to expand slowly toward its original thickness. Finally, after the vibrations stop, a full recovery takes place during stage IV. The shear strength evolves to $\tau_{s s}$, the layer dilates to it's original value (when corrected for compaction due to geometric loss of gouge), and the slip rate returns to the background forcing rate of $10 \mu \mathrm{m} / \mathrm{s}$.

[41] The difference between vibration sets that result in dynamic weakening and those that do not lies in the occurrence of the slip events. When we do not observe stage I, i.e., a large stress drop accompanied by a period of fast slip, then no dynamic weakening takes place (e.g., Figure 8). Strain delocalization, similar to that described by Sleep et al. [2000], accompanies these slip events. Smaller slips that occur during the continued $\sigma_{n}$ vibrations of stage III also appear to be important in the weakening process. Thus we suspect that the earthquakelike slip events, which may reset the state variable to a less developed state, play a significant role in accommodating shear zone weakening.

[42] We note that the strength of the sample during stage III remained approximately constant, independent of the duration of vibrations (e.g., Figure 11c). This observation supports the theory presented by Sleep et al. [2000] that the observed healing during vibrated slide-hold-slide tests is due to a time-dependent process, rather than through mechanical consolidation, as was suggested by Nakatani [2001].

[43] Dynamic weakening appears to depend primarily on the amplitude and frequency of vibrations, rather than the number of vibrations or background creep rate of the shear zone. Figure 12 shows that a small amount of dynamic weakening is predicted at short periods for all loading rates $(1 \leq V \leq 1000 \mu \mathrm{m} / \mathrm{s})$. The model parameters are fixed to the values used in the previous numerical simulations, and again the Dieterich formulation of the rate and state theory systematically predicts the experimental data. The period at which the peak yield strength becomes positive is inversely proportional to $V$.

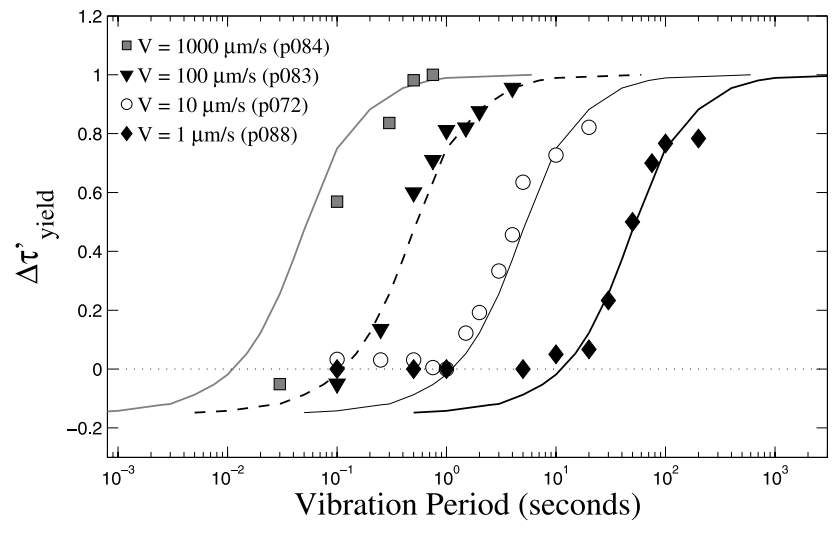

Figure 12. Effect of loading rate and vibration period on frictional strength observed in laboratory data (symbols) and predicted by the Dieterich rate/state friction model (lines). Note that while the theory matched the experimental data at long periods and the systematic variation with velocity, the predicted weakening at short periods was not observed. Model parameters are given in Figure 8 with loading rates of $1-1000 \mu \mathrm{m} / \mathrm{s}$. 


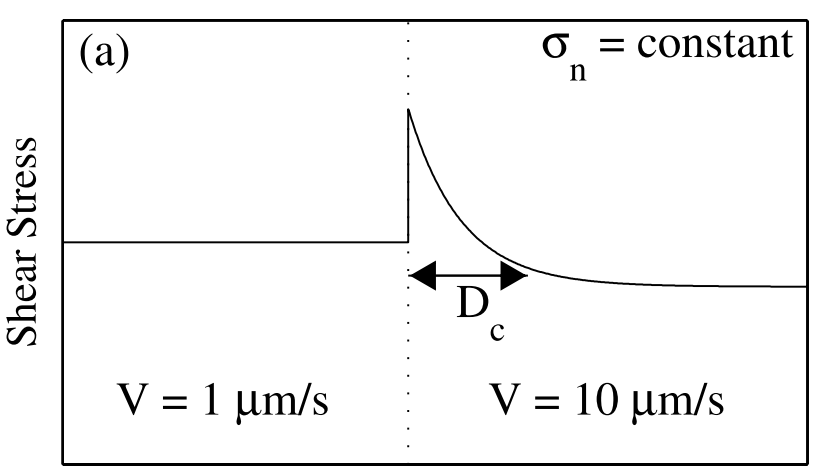

Displacement

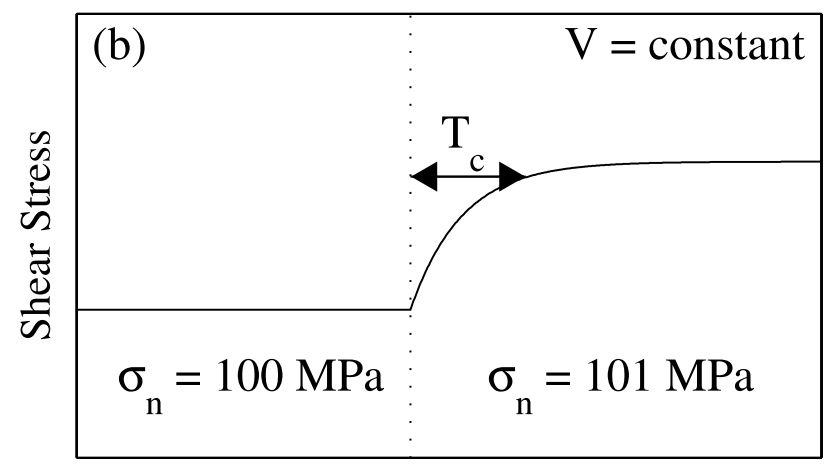

Time

Figure 13. Critical length scales necessary to obtain a steady state frictional strength following a step in $V$ or $\sigma_{n}$. (a) Under constant normal stress conditions, $D_{c}$ is the critical slip distance needed to obtain a new steady state shear strength with a step in $V$. (b) Analogous to $D_{c}, T_{c}$ is a critical time over which the frictional strength will evolve to a new steady state following a step in $\sigma_{n}$, assuming a constant loading rate. $T_{c}$ is thus a critical period, below which the shear zone is continuously evolving as it attempts to respond to changes in $\sigma_{n}$.

The data scatter more at short periods than at long periods. The main discrepancy between data and theory is that the predicted dynamic weakening at short periods is not observed in our experiments. This may be due to inadequacies in the rate and state theory, such as the immediate response to a sudden change in normal stress.

[44] In this study we mainly focus on the destabilizing and weakening effects of vibrations, however, we see from Figure 12 that a common result of vibrations is to strengthen our samples. For most cases, the peak yield strength $\Delta \tau_{\text {yield }}^{\prime}$ is positive, indicating that a dynamic strengthening processes is in affect. This is consistent with the laboratory results of Richardson and Marone [1999], which focused on the healing process. They found that $\sigma_{n}$ vibrations caused increased strengthening during interseismic intervals. We emphasize that under certain conditions $\sigma_{n}$ vibrations increase the absolute strength of a creeping shear zone while others result in dynamic weakening. In particular, high-frequency $\sigma_{n}$ perturbations of sufficiently large amplitude will tempo- rarily reduce the strength of the shear zone, potentially bringing it closer to failure.

\subsection{Resonant Conditions}

[45] The stability of our experimental shear zones is dependent on three parameters: (1) the critical period, $T_{c}$, which was shown by Rice and Ruina [1983] to be

$$
T_{c}=2 \pi \sqrt{a /(b-a)}\left(D_{c} / V\right)
$$

(2) the critical vibration amplitude to destabilize slip, $\epsilon_{c}$, that is approximately [Perfettini et al., 2001]

$$
\epsilon_{c} \approx \frac{b-a}{\mu_{s s}} \frac{1-k / k_{c}}{\sqrt{1+\left(1-\alpha / \mu_{s s}\right)^{2}(b-a) / a}}
$$

and (3) the critical stiffness, $k_{c}$ (see equation (1)). In order for our stiffness-stabilized system $\left(k>k_{c}\right)$ to become unstable, $T / T_{c}$ and $k / k_{c}$ must both be very close to 1.0 , and $\epsilon$ must exceed $\epsilon_{c}$ [Perfettini et al., 2001].

[46] Figure 13 illustrates the meaning of $T_{c}$ in terms of the time necessary for shear strength to evolve to a new steady state level following a single step in normal stress. As expressed in equation (6), $T_{c}$ scales as $D_{c} / V$. Thus for a given loading rate, $T_{c}$ scales directly with $D_{c}$, and both quantities describe a critical length scale necessary to obtain a new shear strength level. When a system is subject to periodic normal stress perturbations, rather than a simple normal stress step, we see that the vibration period strongly controls the response of the system. At vibration periods of $T_{c}$ or longer, frictional strength is able to adjust continuously, allowing the layer thickness and the coefficient of friction to remain constant. Periods below $T_{c}$ result in large-amplitude layer thickness changes that phase lag normal stress oscillations. The layer rapidly dilates and compacts in an attempt to compensate the imposed $\sigma_{n}$ changes. At periods less than $T_{c} / 10$, layer thickness fluctuations allow the shear strength to remain constant while $\sigma_{n}$ rapidly changes. Variations in the layer thickness indicate that interparticle friction can not keep pace with the changes in normal stress. Thus periods less than $T_{c}$, in particular, $T<T_{c} / 10$, are susceptible to instabilities such as the earthquake-like stress drops described above.

[47] Experimentally, $T=T_{c}$ can easily be achieved with the loading rates we used. The critical vibration amplitude $\epsilon_{c}=0.0022$, obtained by inserting the friction parameters used in Figures 8-15 into in equation (7), was exceeded in nearly all of our experiments. However, to achieve unstable resonant behavior, $k / k_{c}$ must be reduced to near 1.0 , and as shown in Figure 14 this is currently unachievable with our testing apparatus.

[48] Figure 14a, a reproduction of Figure 6 in Perfettini et al. [2001], shows the effect of changing $k / k_{c}$ for a set of estimated friction parameters. Figure $14 \mathrm{~b}$ is the same plot calculated using our laboratory measurements as the model parameters with our experimental data (open circles) plotted over the curves. The experimental data follow the lowermost curve, $k / k_{c}=5$. We therefore conclude that the stiffness of our testing apparatus is well above the critical stiffness, and we are unable to attain resonant conditions. Additionally, the calculated frictional response for our 

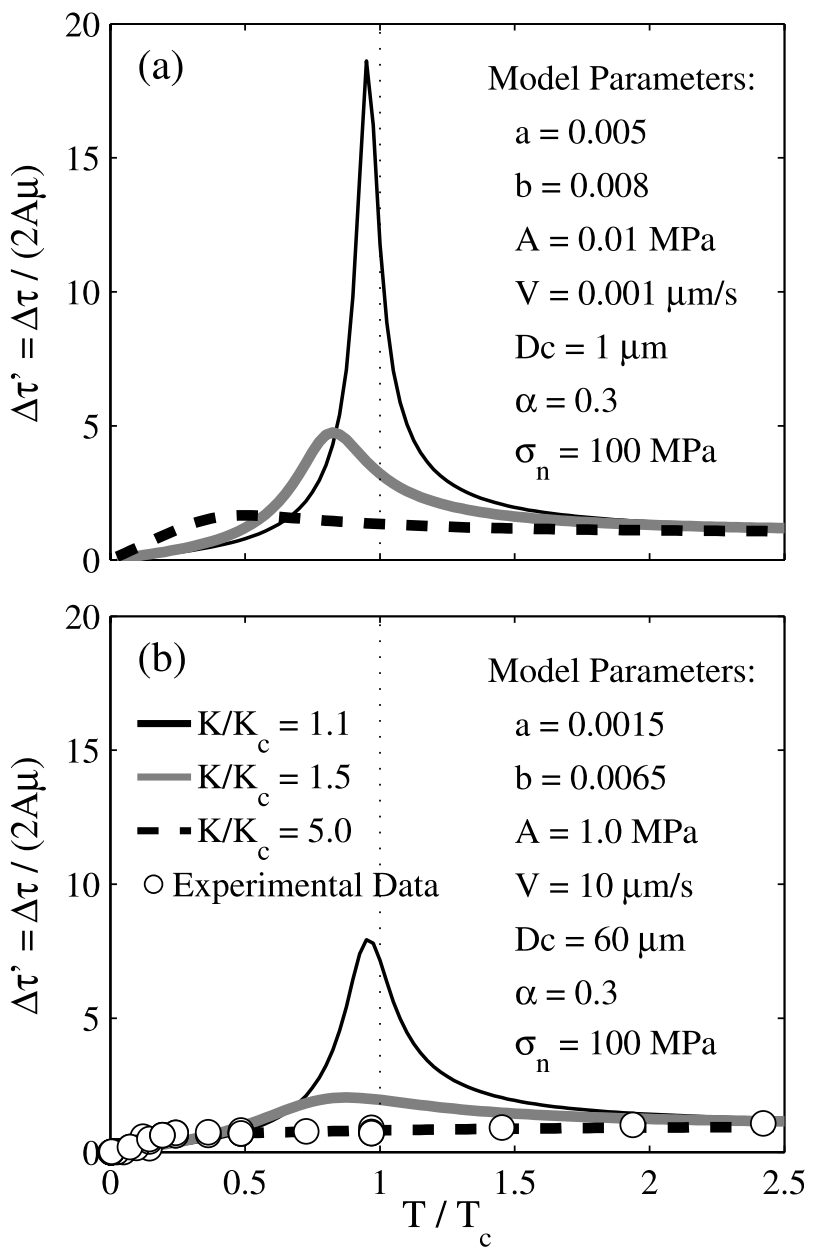

Figure 14. Analytical solutions for the effect of stiffness on frictional strength. (a) Critical parameter values that resulted in a peak response at $T=T_{c}$ [after Perfettini et al., 2001, Figure 6]. (b) Parameters appropriate for our laboratory conditions. Note the reduced amplitude of the resonant peak at $T=T_{c}$ for the laboratory parameters. Superimposed on the calculated strength curves in Figure $14 \mathrm{~b}$ are the experimental data (circles), which approximately follow the $K / K_{c}=5$ curve. Equation (24) of Perfettini et al. [2001] was used to compute the predicted strength values.

laboratory conditions are minimal compared with the expectations for the conditions given in Figure 14a.

[49] While we do not observe resonance, Figure 15 shows that the theoretical critical period, below which the shear strength response is not sufficient to maintain a constant coefficient of friction, is consistent with our observed critical period, below which the shear strength response is completely unable to follow $\sigma_{n}$ vibrations. We find that $T_{c} \approx 10 T_{c}^{*}$, which allows us to extrapolate $T_{c}$ through $T_{c}^{*}$, and thus predict $T_{c}$ for potentially more critical systems (i.e., $k / k_{c} \approx 1$ ).

\subsection{Relevance to Natural Faults}

[50] Making simple assumptions about the friction parameters of natural faults $\left(D_{c}=0.1 \mathrm{~m}\right.$ and $\left.b \approx 2 a\right)$, we can interpret our laboratory results in the context of earth- quake processes. Equation (6) predicts that a critical vibration period of $T=1 \mathrm{~s}$, which is near the microseism peak, will excite a resonant response on a stable fault, slipping at a rate of about a $\mathrm{m} / \mathrm{s}$. Our findings remain the same when the range of assumed values for $a, b$, and $D_{c}$ is broadened to include $1.1 a \leq b \leq 3 a$ and $0.01 \mathrm{~m} \leq D_{c} \leq 1.0 \mathrm{~m}$. Microseismic energy will only excite a resonant response on stable faults sliding at speeds between a few $\mathrm{cm} / \mathrm{s}$ and tens of $\mathrm{m} / \mathrm{s}$, much more rapidly than is typically observed in the Earth.

[51] On a fault creeping at $V=10 \mathrm{~mm} / \mathrm{yr}$, resonance should occur during vibrations with a period of between a few years and a few thousands of years for the range of parameters given above. Stable slip at the rates of rupture propagation may therefore be affected by coseismic vibrations, but tectonic loading rates are too slow to be influenced by high-frequency seismic shaking. Earth tides, which oscillate at a period of 12 hours, may destabilize a fault slipping between $1 \mu \mathrm{m} / \mathrm{s}$ and $0.5 \mathrm{~mm} / \mathrm{s}$; rates intermediate between tectonic loading and coseismic slip. Roy and Marone [1996] estimated that similar slip rates $(1-100 \mathrm{~mm} / \mathrm{s})$ are necessary for a static stress perturbation to trigger inertia-driven motion and nucleate earthquakes. Thus we find that the resonant response to normal stress vibrations does not appear to trigger earthquakes on creeping faults.

[52] Normal-force perturbations appear to have a greater effect on fault strength than stability. Our results show that a fault subject to large-amplitude, short-period vibrations, at $T<0.1 T_{c}$, will exhibit dynamic weakening. Therefore transient stressing from tidal forcing, seismic waves, or other periodic normal stress fluctuations of sufficient amplitude $(\epsilon>0.01)$ with periods less than a few years (for faults creeping at $V=10 \mathrm{~mm} / \mathrm{yr}$ with the friction parameters given above) may all result in fault zone weakening.

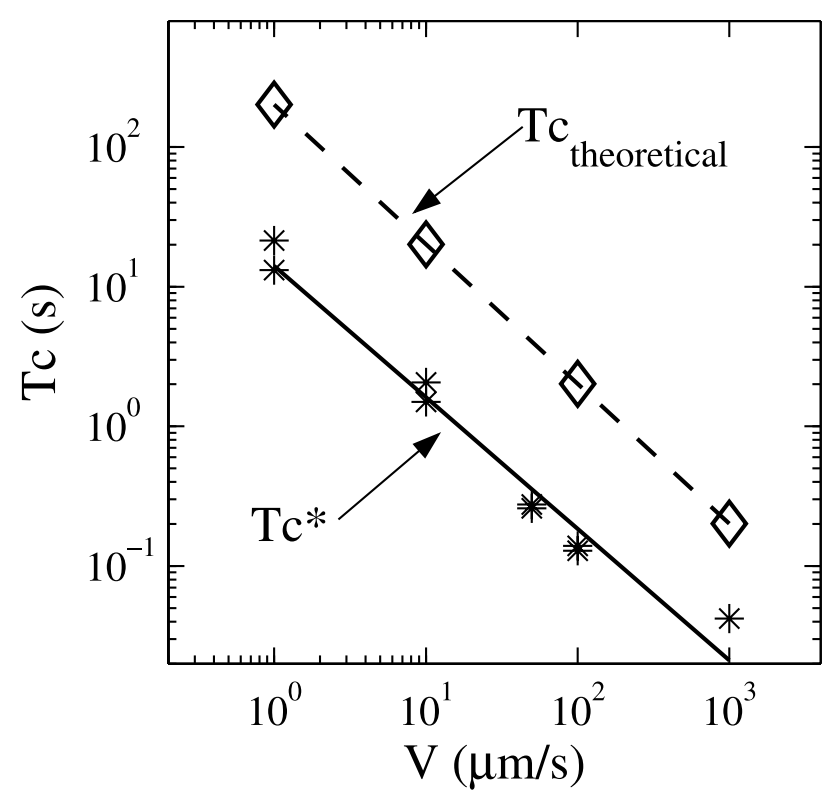

Figure 15. Theoretical definitions of critical period using the friction parameters given in Figure 8 compared with our laboratory-derived values of $T_{c}^{*}$. The relationship, $T_{c} \approx$ $10 T_{c}^{*}$, enabled us to convert $T_{c}^{*}$ to $T_{c}$. 
[53] Some differences are observed between the dynamic weakening process seen in our experiments and the documented cases of dynamic triggering observed in nature. We do not observe a delay in the weakening of our laboratory shear zone, as is suggested by observations of dynamic triggering following the Landers earthquake [Hill et al., 1993]. Gomberg et al. [1997] and Kilb et al. [2002] discuss the possibility of permanent weakening or creation of new fractures due to dynamic stressing. They suggest that "extra" seismicity occurs on new fault surfaces and it may be delayed significantly from the onset of dynamic stressing. No permanent damage from the imposed normal stress vibrations is evident in our experiments, yet our shear zone is not lithified rock and fractures do not form. Sleep et al. [2000] suggest that the observed healing in the slidehold-slide experiments of Richardson and Marone [1999] may be due to the disruption of smooth, sliding surfaces during the imposed vibrations. It is important to note that our experiments are conducted on short timescales and cannot be expected to see long-term, time-dependent weakening or strengthening.

[54] Once the systematics of $\sigma_{n}$ vibrations on creeping shear zones are well determined, it will be advisable to investigate the effect of fluids within the stressed shear zone. Fluid-filled regions such as volcanic and hydrothermal systems are most often affected by dynamic stresses [e.g., Hill et al., 1993; Gomberg and Davis, 1996]. In laboratory experiments conducted by Frye and Marone [2002] enhanced healing is observed in samples with higher water content and the theoretical work of Segall and Rice [1994] shows that the effect of dilatancy on slip instability is strongly influenced by fault zone porosity. It is likely that shaking would cause changes in fluid levels, altering the pore pressure and creating a more critical system.

\section{Summary and Conclusion}

[55] Laboratory experiments testing the effect of normal stress vibrations on steadily creeping shear zones show systematic variation with vibration period, amplitude, and loading rate. Large-amplitude, short-period vibrations result in a small $(\sim 1 \%)$ dynamic weakening of the shear zone that persists throughout the vibrations, with stress drops of $\sim 4.5 \%$ during the onset of vibrations. The weakening is accompanied by dilation and a sharp increase in shear zone slip rate. At periods longer than the critical period, which is a well-defined quantity for each set of experimental conditions, vibrations produce sinusoidal shear strength oscillations that are in phase with the periodic forcing. Small-amplitude vibrations have a negligible effect on mean shear zone strength at all periods. While a resonant response was not observed in our experiments and we do not expect that resonance causes instabilities on many natural faults, we do predict that periodic vibrations will temporarily weaken fault zones.

[56] The Dieterich (aging) friction evolution law reproduces the experimental data more closely than does the Ruina (slip) law. At intermediate and long periods, the Linker and Dieterich formulation for the effect of a step change in normal stress successfully predicts the shear strength behavior, but at short periods no immediate response is observed. It appears that while the Dieterich law together with the Linker and Dieterich parameter predict much of our observed shear strength behavior, they do not adequately account for the effect of high-frequency normal stress vibrations.

[57] Acknowledgments. We would like to thank K. Frye for giving his technical expertise and J. Rice for helpful discussions. This paper benefited from constructive reviews by J. Gomberg, H. Perfettini, and N. Sleep. This research was supported by NSF grant EAR 01-96570 and USGS grant 02HQGR0156, and M.B. was supported by a NSF Graduate Research Fellowship. Contribution 11032 of Woods Hole Oceanographic Institution.

\section{References}

Beeler, N. M., and D. A. Lockner (2003), Why earthquakes correlate weakly with the solid Earth tides: Effects of periodic stress on the rate and probability of earthquake occurrence, J. Geophys. Res., 108(B8), 2391, doi:10.1029/2001JB001518.

Beeler, N. M., T. E. Tullis, and J. D. Weeks (1994), The roles of time and displacement in the evolution effect in rock friction, Geophys. Res. Lett., 21(18), 1987-1990.

Beeler, N. M., T. E. Tullis, M. L. Blanpied, and J. D. Weeks (1996), Frictional behavior of large displacement experimental faults, J. Geophys. Res., 101(B4), 8697-8715.

Bodin, P., S. Brown, and D. Matheson (1998), Laboratory observations of fault-normal vibrations during stick slip, J. Geophys. Res., 103(12), $29,931-29,944$

Brodsky, E. E., E. Roeloffs, D. Woodcock, I. Gall, and M. Manga (2003), A mechanism for sustained groundwater pressure changes induced by distant earthquakes, J. Geophys. Res., 108(B8), 2390, doi:10.1029/ 2002JB002321

Bureau, L., T. Baumberger, and C. Caroli (2000), Shear response of a frictional interface to a normal load modulation, Phys. Rev. E, 62(5), $6810-6820$.

Dieterich, J. H. (1978), Time-dependent friction and the mechanics of stickslip, Pure Appl. Geophys., 116, 790-806.

Dieterich, J. H. (1979), Modeling of rock friction: 1. Experimental results and constitutive equations, J. Geophys. Res., 84(B5), 2161-2168.

Frye, K. M., and C. Marone (2002), The effect of humidity on granular friction at room temperature, J. Geophys. Res., 107(B11), 2309, doi:10.1029/2001JB000654.

Gomberg, J. (1996), Stress/strain changes and triggered seismicity following the $M_{w} 7.3$ Landers, California, earthquake, J. Geophys. Res., 101(B1), 751-764

Gomberg, J., and P. Bodin (1994), Triggering of the $M_{s}=5.4$ Little Skull Mountain, Nevada, earthquake with dynamic strains, Bull. Seismol. Soc. Am. 84(3), 844-853.

Gomberg, J., and S. Davis (1996), Stress/strain changes and triggered seismicity at The Geysers, California, J. Geophys. Res., 101(B1), 733-750.

Gomberg, J., M. L. Blanpied, and N. M. Beeler (1997), Transient triggering of near and distant earthquakes, Bull. Seismol. Soc. Am., 87(2), 294-309.

Harris, R. A. (1998), Introduction to special section: Stress triggers, stress shadows, and implications for seismic hazard, J. Geophys. Res., 103(B10), 24,347-24,358.

Heaton, T. H. (1990), Evidence for and implications of self-healing pulses of slip in earthquake rupture, Phys. Earth Planet. Inter., 64, 1-20.

Hill, D., et al. (1993), Seismicity remotely triggered by the magnitude 7.3 Landers, California, earthquake, Science, 260, 1617-1623.

Karner, S. L., and C. Marone (2001), Frictional restrengthening in simulated fault gouge: Effect of shear load perturbations, J. Geophys. Res., 106(B9), 19,319-19,337.

Kilb, D., J. Gomberg, and P. Bodin (2000), Triggering of earthquake aftershocks by dynamic stresses, Nature, 408, 570-574.

Kilb, D., J. Gomberg, and P. Bodin (2002), Aftershock triggering by complete Coulomb stress changes, J. Geophys. Res., 107(B4), 2060, doi:10.1029/2001JB000202.

Linker, M. F., and J. H. Dieterich (1992), Effects of variable normal stress on rock friction: Observations and constitutive equations, J. Geophys. Res., 97(B4), 4923-4940.

Lockner, D. A., and N. M. Beeler (1999), Premonitory slip and tidal triggering of earthquakes, J. Geophys. Res., 104(B9), 20,133-20,151.

Mair, K., and C. Marone (1999), Friction of simulated fault gouge for a wide range of velocities and normal stresses, J. Geophys. Res., 104(B12), $28,899-28,914$.

Marone, C. (1998), Laboratory derived friction laws and their application to seismic faulting, Annu. Rev. Earth Planet. Sci., 26, 643-696.

Melosh, H. J. (1979), Acoustic fluidization: A new geologic process?, J. Geophys. Res., 84(B13), 7513-7520. 
Nakatani, M. (2001), Conceptual and physical clarification of rate and state friction: Frictional sliding as a thermally activated rheology, J. Geophys. Res., 106(B7), 13,347-13,380.

Perfettini, H., J. Schmittbuhl, J. R. Rice, and M. Cocco (2001), Frictional response induced by time-dependent fluctuations of the normal loading, J. Geophys. Res., 106(B7), 13,455-13,472.

Perfettini, H., J. Schmittbuhl, and A. Cochard (2003), Shear and norma load perturbations on a two-dimensional continuous fault: 2. Dyanmic triggering, J. Geophys. Res., 108(B9), 2409, doi:10.1029/2002JB001805.

Prakash, V. (1998), Frictional response of sliding interfaces subjected to time varying normal pressures, J. Tribology, 120, 97-102.

Rice, J. R., and A. L. Ruina (1983), Stability of steady frictional slipping, J. Appl. Mech., 105, 343-349.

Richardson, E., and C. Marone (1999), Effects of normal stress vibrations on frictional healing, J. Geophys. Res., 104(B12), 28,859-28,878.

Roy, M., and C. Marone (1996), Earthquake nucleation on model faults with rate- and state-dependent friction: Effects of inertia, J. Geophys. Res., 101(B6), 13,919-13,932.

Ruina, A. (1983), Slip instability and state variable friction laws, J. Geophys. Res., 88(B12), 10,359-10,370.

Scholz, C. H. (2003), Good tidings, Nature, 425, 670-671.

Segall, P., and J. R. Rice (1994), Dilatancy, compaction, and slip instability of a fluid-infiltrated fault (abstract), Eos Trans. $A G U, 75(44)$, Fall Meet. Suppl., 425.

Sleep, N. H., E. Richardson, and C. Marone (2000), Physics of friction and strain rate localization in synthetic fault gouge, J. Geophys. Res., 105(B11), 25,875-25,890.

Spudich, P., L. Steck, M. Hellweg, J. Fletcher, and L. Baker (1995), Transient stresses at Parkfield, California, produced by the $M 7.4$ Landers earthquake of June 28, 1992: Observations from the UPSAR dense seismograph array, J. Geophys. Res., 100(B1), 675-690.
Tolstoy, M., F. Vernon, J. Orcutt, and F. Wyatt (2002), Breathing of the seafloor: Tidal correlations of seismicity at Axial volcano, Geology, $30(6), 503-506$

Tworzydlo, W. W., and O. N. Hamzeh (1997), On the importance of normal vibrations in modeling of stick slip in rock sliding, J. Geophys. Res., 102(B7), 15,091-15,103.

Vidale, J. E., and Y.-G. Li (2003), Damage to the shallow Landers fault from the nearby Hector Mine earthquake, Nature, 421, 524-526.

Vidale, J. E., D. C. Agnew, M. J. S. Johnston, and D. H. Oppenheimer (1998), Absence of earthquake correlation with Earth tides: An indication of high preseismic fault stress rate, J. Geophys. Res., 103(B10), 24,56724,572 .

Voisin, C. (2001), Dynamic triggering of earthquakes: The linear slipdependent friction case, Geophys. Res. Lett., 28(17), 3357-3360.

Voisin, C. (2002), Dynamic triggering of earthquakes: The nonlinear slipdependent friction case, J. Geophys. Res., 107(B12), 2356, doi:10.1029/ 2001JB001121.

Wilcock, W. S. D. (2001), Tidal triggering of microearthquakes on the Juan de Fuca Ridge, Geophys. Res. Lett., 28(20), 3999-4002.

Wyss, M., and S. Wiemer (2000), Change in the probability for earthquakes in southern California due to the Landers magnitude 7.3 earthquake, Science, 290, 1334-1338.

M. S. Boettcher, Marine Geology and Geophysics, MIT/WHOI Joint Program, Woods Hole Oceanographic Institution, Woods Hole, MA 02543, USA. (margaret@whoi.edu)

C. Marone, Department of Geosciences, Pennsylvania State University, University Park, PA 16802, USA. (cjm@geosc.psu.edu) 